\title{
Wenn Städte sterben
}

\author{
Württembergische „Statuswüstungen“ des Mittelalters und der Neuzeit
}

Von Nina KüHnle

\section{Vergessene Städte}

Als im Jahr 1962 das von Erich Keyser herausgegebene „Württembergische Städtebuch“ in der Reihe „Deutsches Städtebuch “ erschien ${ }^{1}$, erhielt es in den Literaturbesprechungen der einschlägigen wissenschaftlichen Zeitschriften viel Anerkennung. Das Mammutprojekt hatte, bedingt durch den Zweiten Weltkrieg, eine fast dreißigjährige Bearbeitungszeit in Anspruch genommen und versammelte nun auf 490 Seiten die einheitlich gegliederten Artikel zu insgesamt 169 Städten und drei Stuttgarter Stadtteilen - eine „respektable Leistung“, wie Friedrich Facius in seiner Rezension befand ${ }^{2}$. Hektor Ammann kürte den Band sogleich zu einem „der gelungensten des Gesamtwerkes“ und Hans Jänichen sprach von einem „unentbehrlichen Nachschlagewerk" sowie von der „bedeutendsten Veröffentlichung topographisch-historisch-statistischer Art" seiner Zeit ${ }^{3}$. Trotz der zahlreichen Komplimente wurde jedoch auch Kritik geäußert. Ein gewichtiger Punkt, der sich in beinahe allen Rezensionen wiederfindet, betraf dabei die Auswahl der Stadtporträts. Die Bände der einzelnen Regionen sollten nämlich einen Einblick in das gegenwärtige Städtewesen bieten und hatten daher nur diejenigen Städte berücksichtigt, die zum Bearbeitungszeitpunkt über den entsprechenden Status verfügten. Infolgedessen fanden sehr junge Städte wie Feuerbach und Zuffenhausen, deren Selbständigkeit sich nach der Eingemeindung nach Stuttgart noch dazu schnell erledigt hatte,

1 Württembergisches Städtebuch, hg. von Erich Keyser (Deutsches Städtebuch. Handbuch städtischer Geschichte, Bd.4/2/2), Stuttgart 1962 (künftig: WüSt).

${ }^{2}$ Friedrich Facius, Rezension Württembergisches Städtebuch, in: ZWLG 22 (1963) S. 204-208, hier S. 205. Bei den erwähnten drei Stuttgarter Stadtteilen handelt es sich um die ehemals selbständigen Städte Bad Cannstatt, Feuerbach und Zuffenhausen (WüSt, S.252260).

${ }^{3}$ Hektor Ammann, Rezension Württembergisches Städtebuch, in: Blätter für deutsche Landesgeschichte 98 (1962) S. 417 f., hier S. 417; Hans Jänichen, Rezension Württembergisches Städtebuch, in: Zeitschrift für bayerische Landesgeschichte 26 (1963) S. 747-749, hier S. 749. 
Eingang in das Werk. Ehemalige Städte wie die mittelalterlichen Stadtgründungen Gönningen, Gutenberg oder Hoheneck, die ihren Status im Mittelalter oder in der Neuzeit bereits wieder verloren hatten, sucht man hingegen vergeblich. Als Reaktion darauf führte Hans Jänichen in seiner Besprechung 15 solcher abgegangenen Städte sowie 21 weitere potentielle Kandidaten auf, mit der Mahnung, dass „ohne Beachtung der vielerlei Misch- und Übergangsformen zwischen Dorf- und Stadtgemeinden“ das südwestdeutsche Städtewesen unverständlich bleibe, und auch Friedrich Facius betonte, dass die Forschung „mit vollem Recht“ die Aufnahme dieser Orte gefordert habe ${ }^{4}$; einzig erhört wurde sie nicht. Und so schienen die ehemaligen Städte zu vergessenen Städten zu werden.

\section{Die „Statuswüstung“ - ein Phantom der Stadtgeschichtsforschung}

Dass ehemalige Städte nicht nur in Bezug auf die Bände des „Deutschen Städtebuchs“ dem Vergessen anheim gefallen sind, zeigt sich auch bei aktuellen stadthistorischen Standardwerken. Das inzwischen in stark erweiterter Form vorliegende Kompendium von Eberhard Isenmann etwa, das sich in weiten Teilen an mittelalterlichen Großstädten wie Nürnberg, Köln und Lübeck orientiert, beschränkt sich auf eine knappe Erwähnung „abgekommener“ Städte, die ihre städtische Qualität durch Strukturwandel, Krieg oder Naturkatastrophen eingebüßt hätten ${ }^{5}$. Und bei Evamaria Engel ergeht lediglich der Hinweis, dass das Spätmittelalter von Klein-,

${ }^{4}$ Ebd. (wie Anm. 3) S. 748f. (Zitat auf S. 749); Facius (wie Anm. 2) S. 206. Auch Hektor Ammann macht auf das Fehlen verschwundener Städte aufmerksam (Ammann [wie Anm.3] S. 417). In der „Historischen Zeitschrift“ verzichtete man auf Detailanalysen (Walter SchleSINGER, Rezension Württembergisches Städtebuch, in: Historische Zeitschrift 204 (1967) S. 395-399), während die ZGO gleich ganz auf eine Rezension verzichtete, vielleicht weil Karl Bader bereits dem „Badischen Städtebuch“ (Bd.4/2/1) den Rang eines geschichtswissenschaftlichen Standardwerks abgesprochen hatte (Karl S. BADER, Rezension Badisches Städtebuch, in: ZGO 111 [1963] S.627-631). Auch andere Bände des „Deutschen Städtebuchs" mussten sich die Kritik gefallen lassen, keine ehemaligen Städte aufgenommen zu haben, so das „Städtebuch Rheinland-Pfalz und Saarland“ (Bd. 4/3) bei Ludwig Petry, Stufen und Formen des Städtewesens in Rheinland-Pfalz. Überlegungen im Anschluß an den neuen Band des deutschen Städtebuchs, in: Festschrift Johannes Bärmann, hg. von Ludwig Petry (Geschichtliche Landeskunde, Bd.3), 2 Bde., Wiesbaden 1966-67, Bd.2, S. 1-36, hier S.7. Und das „Bayerische Städtebuch“ (Bd.5), das nur (oder immerhin) zwei ehemalige Städte berücksichtigt hatte, bei Adolf LAYER, Kurzlebige mittelalterliche Stadtgründungen im östlichen Schwaben, in: Zeitschrift des Vereins für Schwaben und Neuburg 69 (1975) S.7-17, hier S. 7. Eine generelle Kritik zur Auswahlpraxis des „Deutschen Städtebuchs“ findet sich bei Evamaria Engel, Ehemalige Städte, Städtchen und Flecken im Land Brandenburg, in: Jahrbuch für brandenburgische Landesgeschichte 52 (2001) S. 7-29, hier S. 7.

5 Eberhard Isenmann, Die deutsche Stadt im Mittelalter 1150-1550. Stadtgestalt, Recht, Verfassung, Stadtregiment, Kirche, Gesellschaft, Wirtschaft, Wien/Köln/Weimar 2012, hier S. 55 . 
Kleinst- und Minderstädten geprägt gewesen sei ${ }^{6}$. Dabei zeigt schon Hans Jänichens Auflistung für den heutigen württembergischen Raum die hohe Relevanz des Themas ${ }^{7}$, das freilich kein genuin württembergisches Phänomen darstellt, sondern auch in den Untersuchungen anderer Regionen immer wieder begegnet. Entsprechend lang ist die Liste ehemaliger Städte, die sich geographisch von der Nordostschweiz (z.B. Weesen, Fürstenau und Biberstein), der Westschweiz (z.B. Vuippens, La Tour-de-Trême und Vaulruz) und dem östlichen Schwaben (z. B. Babenhausen, Neuburg an der Kammel und Ottobeuren) über die Südpfalz (z.B. Rheinzabern), Niedersachsen (z. B. Rosenthal bei Peine, Aerzen und Wallensen) und Westfalen (z. B. Lüdinghausen und Alme) bis hin zu Brandenburg (z.B. Fürstenwerder und Jagow) erstreckt ${ }^{8}$. Trotz dieser Fülle aber ist die Erforschung sol-

${ }^{6}$ Evamaria Engel, Die deutsche Stadt im Mittelalter, München 1993, hier S. 36.

7 Vgl. auch Herbert RAIsch, Die Zwergstädte Württembergs. Begriff und Verbreitung, in: Berichte zur deutschen Landeskunde 40 (1968) S. 36-58, hier S. 50-52. Schon die ältere Forschung hat auf ehemalige Städte aufmerksam gemacht: Karl Weller, Besiedlungsgeschichte Württembergs vom 3. bis 13. Jahrhundert (Besiedlungsgeschichte Württembergs, Bd.3), Stuttgart 1938, hier S.352; Viktor ERNST, Die Entstehung der württembergischen Städte, in: Württembergische Studien zum 70. Geburtstag von Eugen Nägele, hg. von Peter Goessler, Stuttgart 1926, S. 121-137, hier S. 133.

${ }^{8}$ Zur Nordostschweiz s. Martina Stercken, Städte der Herrschaft. Kleinstadtgenese im habsburgischen Herrschaftsraum des 13. und 14. Jahrhunderts (Städteforschung, Bd. A 68), Köln/Weimar/Wien 2006, hier S. 39-41; Hektor AmmanN, Die schweizerische Kleinstadt in der mittelalterlichen Wirtschaft, in: Festschrift Walther Merz, Aarau 1928, S.158-215, hier S. 182; Ders., Zwei unbekannte mittelalterliche Städte der Waadt. Ein Beitrag zum Problem des Verschwindens der mittelalterlichen Städte unseres Landes, in: Mélanges d'histoire économique et sociale en hommage au professeur Antony Babel, 2 Bde., Genf 1963, Bd.1, S. 72 93, hier S. 76-79. Ammann erwähnt, dass von insgesamt 93 Stadtverlusten in der Nordostschweiz in 45 Fällen der Stadtstatus verloren ging (ebd., S. 75 f.). Speziell zu Weesen im Kanton St. Gallen s. ebd., S. 76; Martina Stercken, Stadtstatus und zentralörtliche Funktion. Weesen am Walensee als habsburgische Kleinstadt und Flecken unter schwyzerischer und glarnerischer Herrschaft, in: Siedlungsforschung 11 (1993) S. 219-236. Zur Westschweiz s. Roland Flückiger-SeILER, Die Basse-Gruyère. Entstehung und Untergang einer überfüllten Städtelandschaft des Spätmittelalters in der Westschweiz, in: Siedlungsforschung 11 (1993) S. 167-199, hier v. a. S. 170 und 172. Zu Ostschwaben s. LAyer (wie Anm. 4). Zur Südpfalz s. Petri (wie Anm. 4) S.7 mit Anm. 19. Zu Niedersachsen s. Thomas Küntzel, Stadtund Marktwüstungen im Braunschweiger Land: ein systematischer Ansatz, in: Salzgitter-Jahrbuch 30 (2012) S.35-60, hier S.35-37; Hans-Georg Stephan, Dynastische Städtegründungen, Märkte, abgesunkene mittelalterliche Städte und Stadtwüstungen im braunschweigischen Weserbergland: die Grafen von Dassel und die Grafen von Everstein im Kampf um die Landesherrschaft mit den Welfen im späteren Herzogtum Braunschweig und in benachbarten Gebieten, in: Salzgitter-Jahrbuch 30 (2012) S. 61-156, hier S. 83-85 und 108f. Zu Westfalen s. Carl HaAse, Die Entstehung der westfälischen Städte (Veröffentlichungen des Provinzialinstituts für Westfälische Landes- und Volkskunde, Bd. 1/11), Münster ${ }^{2} 1965$, hier S. 132 und 161. Zu Brandenburg s. EngEL (wie Anm. 4) S. 13 f.; Lieselott ENDERs, Werden und Vergehen kleinerer Städte während des Spätmittelalters und der frühen Neuzeit. Funktions- und Existenzbedingungen in der Mark Brandenburg, in: Siedlungsforschung 11 (1993) S. 111-122, hier S. 113 f.; Thomas Schenk, Werden, Vergehen, Werden ... Zur Stadt Freyenstein in der Ostprignitz, in: Wie die Mark entstand. 850 Jahre Mark Bran- 
cher Orte noch nicht über die Stufe exemplarischer Studien hinausgekommen. Stattdessen zeigt sich bereits in der Namengebung die ganze Unsicherheit im Umgang mit diesen anscheinend nicht recht greifbaren Erscheinungen, die wahlweise als „ehemalige“, „abgegangene“, „abgekommene“, „gewesene“, „abgesunkene“ oder „ausgegangene“ Städte sowie in inhaltlich wertender Weise als „Fehlgründungen“ bezeichnet werden ${ }^{9}$. Zwar gibt es, mit Ausnahme der „Fehlgründung“, an

denburg. Fachtagung unter der Schirmherrschaft von Gunter Fritsch, Präsident des Landtages Brandenburg, vom 20. bis 22. Juni 2007 in Brandenburg an der Havel, hg. von Thomas Kersting, Wünsdorf 2009, S. 216-224, hier S. 216. Schenk verweist auf die Zahl von ca. 50 ehemaligen Städten in Brandenburg (ebd.).

9 „Ehemalige“ Stadt bei Engel (wie Anm. 4); Schenk (wie Anm. 8) S. 216. „Abgegangene" Stadt bei Heinz Sтоов, Minderstädte. Formen der Stadtentstehung im Spätmittelalter (1959), in: Ders., Forschungen zum Städtewesen in Europa, Bd.1: Räume, Formen und Schichten der mitteleuropäischen Städte. Eine Aufsatzfolge, Köln/Wien 1970, S. 225-245, hier S. 241. „Abgekommene“ Stadt bei Isenmann (wie Anm. 5) S. 55. „Gewesene“ Stadt z.B. bei Layer (wie Anm.4) S. 7. „Abgesunkene“ Stadt bei Meinrad Scha ab, Städtlein, Burg-, Amts- und Marktflecken Südwestdeutschlands in Spätmittelalter und früher Neuzeit, in: Zentralität als Problem der mittelalterlichen Stadtgeschichtsforschung, hg. von Emil MeYNEN (Städteforschung, Bd. A 8), Köln/Wien 1979, S. 219-271, hier S. 248. „Ausgegangene“ Stadt und „Fehlgründung“ bei HaAse (wie Anm. 8) S.60. „Fehlgründung“ bei Wolfgang EgGert, Städtenetz und Stadtherrenpolitik. Ihre Herausbildung im Bereich des späteren Württemberg während des 13. Jahrhunderts, in: Stadt und Städtebürgertum in der deutschen Geschichte des 13. Jahrhunderts, hg. von Bernhard Töpfer (Forschungen zur mittelalterlichen Geschichte, Bd. 24), Berlin 1976, S. 108-228, hier S. 228; Edith Ennen, Die europäische Stadt des Mittelalters (Sammlung Vandenhoeck), Göttingen ${ }^{4} 1987$, S. 105. Es finden sich zudem die Varianten „Fehlkalkulation“ sowie „missglückte Gründung“ bei Jürgen Sydow, Städte im deutschen Südwesten. Ihre Geschichte von der Römerzeit bis zur Gegenwart, Stuttgart/Berlin/Köln/Mainz 1987, S. 112; Franz Irsigler, Städtelandschaften und kleine Städte, in: Städtelandschaften in Altbayern. Studien zum Phänomen der Kleinstädte während des Spätmittelalters und der Frühen Neuzeit, hg. von Helmut Flachenecker/Rolf KiessLing (Zeitschrift für bayerische Landesgeschichte. Beihefte, Bd. B 15), München 1999, S. 13-38, hier S.28. Der negativ wertende, erst aus der Rückschau herrührende Ausdruck „Fehlgründung“ stieß auf vielfache Kritik und ließ Bernd Ulrich Hucker die berechtigte Frage stellen: „Wie viele Jahrhunderte Existenz muß eine mittelalterliche Stadt aufbringen, um in der Retrospektive als, richtig' geplant gelten zu dürfen?" (Bernd Ulrich Hucker, Die untergegangene Bergstadt Blankenrode im Diemel-Eder-Kupfererzrevier. Beobachtungen zum Problem abgetragener Bergstädte, in: Montanwirtschaft Mitteleuropas vom 12. bis 17. Jahrhundert. Stand, Wege und Aufgaben der Forschung, hg. von Werner Kroker/Ekkehard Westermann [Der Anschnitt. Beiheft, Bd. 2], Bochum 1984, S.103-110, hier S.108). Vgl. außerdem Enders (wie Anm. 8) S. 120; Schenk (wie Anm. 8) S. 216; Hans-Georg Stephan, Stadtwüstungen in Mitteleuropa. Ein erster Überblick, in: Medieval Europe Brugge 1997. International Conference of Medieval and Later Archaeology. 1.-4. October 1997, Bd. 1: Urbanism in Medieval Europe, hg. von Guy DeBoe (IAP rapporten, Bd. 1), Zellik 1997, S. 329360, hier S. 330; Arnold Scheuer brandt, Südwestdeutsche Stadttypen und Städtegruppen bis zum frühen 19. Jahrhundert. Ein Beitrag zur Kulturlandschaftsgeschichte und zur kulturräumlichen Gliederung des nördlichen Baden-Württemberg und seiner Nachbargebiete (Heidelberger Geographische Arbeiten, Bd. 32), Heidelberg 1972, S. 112. Vgl. insgesamt auch Thomas Küntzel, Stadtwüstungen des Mittelalters und der Neuzeit: Formen, Ursachen, Perspektiven, in: Schrumpfende Städte. Ein Phänomen zwischen Antike und Moderne, hg. 
all diesen Umschreibungen nichts auszusetzen - sie finden sich zum Teil auch in diesem Beitrag wieder -, jedoch erscheint das Ringen um Begrifflichkeiten untypisch für eine Stadtgeschichtsforschung, die bislang mit großer Lust am Definieren und Typologisieren den unterschiedlichen Stufen von Urbanität nachspürte.

Schon ein oberflächlicher Blick in einschlägige stadthistorische Untersuchungen offenbart ein breites Spektrum verschiedenster urbaner und präurbaner Kategorien. So unterscheidet man, abgesehen von der Stadt als solcher, die je nach angesetztem Kriterium als Groß-, Mittel- oder Kleinstadt, als Reichsstadt, freie Stadt oder Territorialstadt, als Handelsstadt, Bergstadt oder Ackerbürgerstadt etc. gefasst werden $\mathrm{kann}^{10}$, unter anderem die in entsprechenden Quellen vorkommenden Klassifizierungen Flecken, Märkte, Weichbilder, Freiheiten und Täler. Der Flecken wird dabei als eine Verbindung von einer Burg oder einem Markt mit einer Siedlung verstanden, der Markt als Übergangsform zwischen Stadt und Dorf, die vor allem in Südböhmen, Österreich und Bayern verbreitet ist, das Weichbild als kleinerer nichtstädtischer Ort im niedersächsisch-westfälischen Raum, die Freiheit als unbefestigte südwestdeutsche Zwischenform und das Tal als präurbane Variante am nördlichen Oberrhein und am Mittelrhein ${ }^{11}$. Hinzu gesellt sich der quellenmäßig gleichfalls fassbare Begriff des Städtleins, der nach Meinrad Schaab diejenigen Städte im neuzeitlichen Württemberg markierte, die ihre administrative Funktion als Oberamtssitz verloren hatten - eine Definition, die sich auf das mittelalterliche Württemberg allerdings noch nicht anwenden lässt, das noch keine Oberämter kannte $^{12}$. Heinz Stoob hat die Formenvielfalt von Flecken, Markt, Freiheit, Weichbild und Tal unter dem Konzept der Minderstadt subsumiert und wollte darunter unterentwickelte, vom Landesherrn ganz bewusst und von vorneherein reduzierte städtische Siedlungen verstanden wissen, die nicht mehr Dorf, aber auch noch nicht Vollstadt seien. Sein Versuch der Vereinfachung stieß jedoch auf sehr geteilte Mei-

von Angelika Lampen/Armin Owzar (Städteforschung, Bd.A 76), Köln/Weimar/Wien 2008, S. 109-144, hier S. 112.

10 So z. B. bei Isenmann (wie Anm. 5) S. 56-58, 62f. und 281-285.

11 Zum Flecken s. z.B. Sснан (wie Anm. 9) S.228f. und 232; Clausdieter Sснотт, Zwischen Dorf und Stadt. Das Flecken. Das Beispiel Zurzach, in: Das Recht im kulturgeschichtlichen Wandel. Festschrift für Karl Heinz Burmeister zur Emeritierung, hg. von Bernd Marquardt, Konstanz 2002, S. 283-303. Zum Markt s. z.B. Walter Schlesinger, Der Markt als Frühform der deutschen Stadt, in: Vor- und Frühformen der europäischen Stadt im Mittelalter. Bericht über ein Symposium in Reinhausen bei Göttingen vom 18. bis 24. April 1972, hg. von Herbert Jankunn/Walter Schlesinger/Heiko Steuer (Abhandlungen der Akademie der Wissenschaften in Göttingen. Philosophisch-historische Klasse, Bd. 3/8384), Göttingen 1973-74, Bd. 1, S. 262-293; Sтоов (wie Anm. 9) S. 234-236. Zum Weichbild s. ebd., S. 226f. Zur Freiheit s. ebd., S. 229-231; Ennen (wie Anm. 9) S. 105. Zum Tal s. Sтоов (wie Anm. 9) S. 232; SchaA (wie Anm. 9) S. 232.

12 Ebd., S. 223-227 (hier auch Beispielstädte, die im Mittelalter noch durchweg als Stadt tituliert werden und erst später den Beinamen Städtlein erhalten). Vgl. auch Walter Grube, Vogteien, Ämter, Landkreise in Baden-Württemberg, Bd.1: Geschichtliche Grundlagen, Stuttgart 1975, S. 14f.; Sтоов (wie Anm. 9) S.232f. 
nung, da der entscheidende Punkt, die Intention des (Minder-)Stadtherrn nämlich, in den meisten Fällen kaum rekonstruierbar ist ${ }^{13}$. Den Begriffsdschungel komplettiert schließlich noch das Stadtdorf, das als Zwitterform ein mit Stadtrecht ausgestattetes Dorf mit maximal 200 Einwohnern meint ${ }^{14}$.

Die vielen Beispiele in Verbund mit einer wahren Flut an wissenschaftlichen Untersuchungen machen deutlich, dass das Interesse der Stadtgeschichtsforschung an den einzelnen Stufen zwischen Dorf und Stadt ungebrochen ist und erste Ansätze der systematischen Erfassung klare Zuordnungen und Benennungen ermöglichen sollen. Gleichwohl lassen sich gerade die hier zu betrachtenden ehemaligen Städte nicht in diese klaren Strukturen einpassen. Denn allen Definitionen vom Flecken bis zum Stadtdorf liegen statische Konzepte zugrunde, Schubladen gewissermaßen, wohingegen im Sinngehalt unserer Fälle dynamisch-transformatorische Elemente vorherrschend sind - und zwar nicht in progressiver Weise wie bei den Prozessen der Stadtwerdung etwa, sondern in regressiver. Es mag daher wenig verwundern, dass gerade in der vornehmlich von Siedlungsgeographen und Archäologen dominierten Wüstungsforschung brauchbare Paradigmen entwickelt wurden. Hatte man sich hier anfänglich noch traditionell mit Dorfwüstungen beschäftigt, trat seit den 1960er-Jahren der seltenere Fall der Stadtwüstung in den Vordergrund, womit bisweilen, etwa für Landsberg bei Wolfhagen (Landkreis Kassel), Nienover (Landkreis Northeim) oder Freyenstein in der Prignitz (heute zur Stadt Wittstock/Dosse im Landkreis Ostprignitz-Ruppin gehörig), umfangreiche archäologische Untersuchungen verbunden waren ${ }^{15}$. Im Zuge dessen hat der

13 Ebd., S. 241-245; Engel (wie Anm. 6) S. 36; Edith Ennen, Die sog. „Minderstädte“ im mittelalterlichen Europa, in: DiEs., Gesammelte Abhandlungen zum europäischen Städtewesen und zur rheinischen Geschichte, 2 Bde., Bonn 1977/87, Bd.2, S. 70-85, hier bes. S. 70 und 85. Vgl. auch Irsigler (wie Anm. 9) S. 28-30. Eberhard Isenmann bezeichnet die Zeitspanne von 1300-1450 als „Zeit der Minderstädte“, obwohl er auch auf die Kritik am Begriff aufmerksam macht (Isenmann [wie Anm. 5] S. 54f.). Kritik übt auch Haase, der die Unterscheidung zwischen Minderstadt und Kümmerform nicht gegeben sieht und als Minderstadt in weiterem Sinne nur nicht mehr voll entwickelte Städte versteht (HAASE [wie Anm.8] S.258).

${ }^{14}$ Felicitas Schmieder, Die mittelalterliche Stadt (Geschichte kompakt), Darmstadt 2005, S. 94.

${ }^{15}$ Stellvertretend für die klassische Wüstungsforschung s. z.B. Wilhelm AвEL, Die Wüstungen des ausgehenden Mittelalters (Quellen und Forschungen zur Agrargeschichte, Bd. 1), Stuttgart ${ }^{2} 1955$; Ulrich GuYAN, Die mittelalterlichen Wüstungen als archäologisches und geographisches Problem, in: Schweizerische Zeitschrift für Geschichte 26 (1946) S. 433-478; Peter Rückert, Landesausbau und Wüstungen des hohen und späten Mittelalters im fränkischen Gäuland (Mainfränkische Studien, Bd.47), Würzburg 1990; Ingeborg VEITH, Wüstungen im Neckarland und auf der Schwäbischen Alb, Diss. masch. Tübingen 1957; Dietrich Weber, Die Wüstungen in Württemberg. Ein Beitrag zur historischen Siedlungs- und Wirtschaftsgeographie von Württemberg (Stuttgarter Geographische Studien, Bd. A 4/5), Stuttgart 1927. Zu den Stadtwüstungen vgl. Küntzel (wie Anm. 8); Ders. (wie Anm. 9); Stephan (wie Anm.9); Ders. (wie Anm.8) v.a. S.76-109; Thomas Küntzel, Die Stadtwüstung Nienover im Solling. Auswertung der Befunde zur Stadttopographie, Hausbau und Stadtbe- 
Mittelalterarchäologe Thomas Küntzel in Anlehnung an Kurt Scharlau, Hans Mortensen und Wilhelm Abel eine Systematik für Stadtwüstungen entworfen, bei der er neben der vollständigen (etwa in Folge einer Siedlungsverlegung) und der partiellen Stadtwüstung auch funktionale Wüstungen und „Statuswüstungen“ unterscheidet. Die funktionale Wüstung ist dabei durch einen Verlust städtischer Funktionen, nicht aber des Stadtstatus gekennzeichnet, während die „Statuswüstung" die ehemalige Stadt meint. Beide Formen können durchaus als einzelne Stufen des Wüstungsvorgangs verstanden werden, die bisweilen aufeinander folgten. So ging der „Statuswüstung“ meist eine „Funktionswüstung“ voraus, wie noch zu sehen sein wird. Meinrad Schaabs Definition des Städtleins hingegen hat gezeigt, dass eine Stadt auch nach dem Verlust ihrer administrativen Kompetenz weiterexistieren konnte, als Funktionswüstung folglich ohne zwingenden Wegfall des Stadtstatus ${ }^{16}$.

Unsere ehemaligen Städte sind also in diesem terminologischen Sinne als „Statuswüstungen“ anzusprechen. Und wie viele es davon gibt, haben bereits die anfänglich erwähnten Beispiele angedeutet. Entsprechend hat schon Heinz Stoob gefordert, dass aufgrund der nicht zu überblickenden Dunkelziffer abgegangener Städte eine „zusammenfassende Darstellung“ dringend notwendig sei ${ }^{17}$. Dies kann der vorliegende Beitrag freilich nicht leisten. Wir wollen uns im Folgenden aber, der Kritiken am „Württembergischen Städtebuch“ eingedenk, einer Reihe von württembergischen „Statuswüstungen“ des Mittelalters und der Neuzeit zuwenden, um in vergleichender Analyse die Charakteristika dieser Fälle wie auch der damit zusammenhängenden Prozesse zu erarbeiten.

festigung im 13. Jahrhundert (Materialhefte zur Ur- und Frühgeschichte, Bd.40), Raden 2009; Christa Plate, Stadtwüstungen in den Bezirken Potsdam und Frankfurt (Oder), in: Archäologische Stadtkernforschung in Sachsen. Ergebnisse - Probleme - Perspektiven, hg. von Heinz-Joachim Vogt (Arbeits- und Forschungsberichte zur Sächsischen Bodendenkmalpflege. Beiheft, Bd.19), Berlin 1990, S.197-215; Schenk (wie Anm. 8). Nach Thomas Schenk sind bislang 60 echte Stadtwüstungen bekannt (ebd., S. 216).

${ }^{16}$ Zur Wüstungssystematik s. KüntZEL (wie Anm. 9) S. 118f. mit Schaubild 2 auf S. 119; Ders. (wie Anm. 8) S.37f. Vgl. auch Stephan (wie Anm. 9) S. 329-332. Der von Thomas Küntzel vorgeschlagene Begriff der „Statuswüstung“, mit dem im Folgenden operiert wird, zielt, anders als die Termini der traditionellen Wüstungsforschung, nicht auf Prozesse der spätmittelalterlichen Siedlungs- und Bevölkerungsrückgänge, sondern auf die gänzlich anders gelagerten Verluste städtischer Qualität. Da er sowohl die Wüstungs- als auch die Stadtgeschichtsforschung um neue Aspekte zu bereichern vermag, ist seine baldige wissenschaftliche Diskussion und Implementierung wünschenswert. Bis dahin wird sein Vorschlagscharakter durch Anführungszeichen gekennzeichnet.

17 Стоов (wie Anm. 9) S. 241. Gleiches fordert übrigens Hans-Georg Stephan für die Archäologie: Stephan (wie Anm.9) S.329f. Thomas Küntzel beziffert die Zahl städtischer Wüstungen (sowohl der vollständigen als auch der partiellen) auf immerhin 10-20\% des städtischen Gesamtbestandes (KüNTZEL [wie Anm. 9] S. 110). 


\section{Württembergische „Statuswüstungen“ des Mittelalters und der Neuzeit}

Da das spätmittelalterliche Württemberg als städtereichstes Territorium Südwestdeutschlands gilt ${ }^{18}$, überrascht es wenig, dass Hans Jänichen ganze 36 „Statuswüstungen“ im heutigen württembergischen Raum in Aussicht stellt. Für die folgende Untersuchung wurden acht Beispiele ausgewählt, die bereits zu einem frühen Zeitpunkt ihrer Stadtgeschichte an die mittelalterliche Grafschaft Württemberg kamen und entweder noch im Mittelalter oder aber in der Neuzeit ihre städtische Qualität einbüßten. Es sind dies Gönningen (heute Stadtteil von Reutlingen), Gutenberg (seit 1975 Teil der Gemeinde Lenningen), Heiningen (Landkreis Göppingen), Hohenhaslach (heute Stadtteil von Sachsenheim), Horrheim (heute Stadtteil von Vaihingen an der Enz), Hoheneck (heute Stadtteil von Ludwigsburg), Neustadt (heute Stadtteil von Waiblingen) und Ochsenburg (seit 1975 Teil der Gemeinde Zaberfeld) ${ }^{19}$.

Für die stadtgeschichtliche Forschung sind mit der Untersuchung von „Statuswüstungen“ gleich mehrere wichtige Aspekte verbunden, die denn auch als Leitlinien für die folgende Analyse dienen sollen. Als buchstäbliche Übergangsform vom Dorf zur Stadt und wieder zurück machen ehemalige Städte nicht nur die Stadtwerdungsprozesse, sondern auch die entsprechenden rückläufigen Entwicklungen (be-)greifbar und führen somit an die Frage heran, was überhaupt verloren gehen muss, um keine Stadt mehr zu sein, bzw. worin also das spezifisch Städtische einer Stadt besteht ${ }^{20}$. Zudem komplettieren „Statuswüstungen“ als weiterer Mosaikstein das weite Feld der urbanen und präurbanen Abstufungen und tragen so nicht nur zum Verständnis des südwestdeutschen Städtewesens bei, wie Hans Jänichen zu bedenken gab, sondern des Städtewesens im Allgemeinen. Der gemein-

18 Volker Trugenberger, Ob den portten drey hirschhorn in gelbem veld-Die württembergische Amtsstadt im 15. und 16. Jahrhundert, in: Landesherrliche Städte in Südwestdeutschland, hg. von Jürgen Treffeisen/Kurt Andermann (Oberrheinische Studien, Bd. 12), Sigmaringen 1994, S. 131-156, hier S. 131. Vgl. auch Rudolf SEIGEL, Die württembergische Stadt am Ausgang des Mittelalters. Probleme der Verfassungs- und Sozialstruktur, in: Die Stadt am Ausgang des Mittelalters, hg. von Wilhelm Rausch (Beiträge zur Geschichte der Städte Mitteleuropas, Bd. 3), Linz 1974, S. 177-193, hier S. 177.

19 Vgl. Das Land Baden-Württemberg. Amtliche Beschreibung nach Kreisen und Gemeinden, hg. von der Landesarchivdirektion Baden-Württemberg, 8 Bde., Stuttgart 19741983 (künftig: LBW), Bd.7, S.63f. (Gönningen), Bd.3, S. 200 (Gutenberg), Bd.3, S. $334 \mathrm{f}$. (Heiningen), Bd.3, S. 426 (Hoheneck), Bd.3, S. 449 f. (Hohenhaslach), Bd.3, S. 463 (Horrheim), Bd.3, S. 561 f. (Neustadt) und Bd. 4, S. 87 (Ochsenburg). Weitere Literatur folgt unten. Vgl. auch die Karte bei Seigel (wie Anm. 18) S. 178. Auf das bei Seigel eingezeichnete weitere Beispiel Asperg wurde verzichtet, da es der Stadt, nach dem Verlust ihres Stadtrechts 1714, im Gegensatz zu den anderen Beispielen gelungen war, selbiges im Jahr 1875 wiederzuerlangen (LBW, Bd. 3, S. 376-378).

20 Vgl. auch KüntZel (wie Anm. 9) S. 127: „Das Auftreten von Stadtwüstungen steht in unmittelbarem Zusammenhang mit der generellen Stadtentwicklung und kann den inneren Charakter dieses Vorgangs zusätzlich beleuchten." 
same territoriale Kontext der Fallbeispiele hat darüber hinaus den Vorteil, dass die acht „Statuswüstungen“ in ihren Gemeinsamkeiten und Unterschieden noch besser vergleichbar sind und einen Einblick in die württembergische Urbanisierungsgeschichte gewähren. In Anbetracht des großen Städtereichtums nämlich ist zu untersuchen, warum gerade die ausgewählten Orte auf der Strecke blieben und welche Faktoren diese Vorgänge begünstigten.

Der erste Analyseschritt widmet sich daher zunächst der Stadtwerdung und ihren Begleitumständen, um die Voraussetzungen und Möglichkeiten der späteren „Statuswüstungen“ zu veranschaulichen. Eng damit verbunden sind die Motive der Stadtgründer, die ihre jungen Städte nur kurze Zeit begleiteten. Der Übergang ins württembergische Territorium markierte einen entscheidenden Einschnitt für die städtische Entwicklung, und es wird zu prüfen sein, inwiefern sich Rahmenbedingungen änderten und was dies für unsere Beispiele bedeutete. Der letzte Abschnitt beschäftigt sich schließlich mit dem Verlust des Stadtstatus und beleuchtet sowohl die äußerlichen Anzeichen als auch die Ursachen des Prozesses.

\section{Von der Siedlung zur Stadt: Prozesse der Stadtwerdung}

Am 27. August 1284 stellte König Rudolf von Habsburg eine Urkunde aus, die dem Ort Heiningen besondere Privilegien zuerkannte. Das Dorf erhielt die Verfassung, die Rechte und die Freiheiten Freiburgs im Breisgau und durfte künftig einen Wochenmarkt abhalten, dessen Besuchern Sicherheit und Freiheit garantiert wurden ${ }^{21}$. War Heiningen damit zur Stadt erhoben worden?

Wann eine Siedlung zur Stadt wurde, ist seit jeher eine der am intensivsten diskutierten Fragen in der Stadtgeschichtsforschung und am konkreten Beispiel mangels Quellen meist nur unzureichend oder näherungsweise feststellbar. Das fast gänzliche Fehlen von Gründungsurkunden, das sich in seiner Regelhaftigkeit durch Dokumentenverluste allein nicht erklären lässt, hat die berechtigte Frage aufgeworfen, ob solche Schriftstücke überhaupt existierten ${ }^{22}$. Doch auch in den seltenen Fällen, in denen sich Rechtsverleihungen erhalten haben, kann man nicht automatisch auf deren Realisierung schließen. So wurde zwar die Gültigkeit der Heininger Urkunde daran festgemacht, dass ihr Wortlaut nahezu identisch sei mit dem Privileg von Sulz am Neckar ${ }^{23}$, das ohne jeden Zweifel zur Stadt aufstieg, je-

21 Wirtembergisches Urkundenbuch, hg. von dem Königlichen Staatsarchiv in Stuttgart, 11 Bde., Stuttgart 1849-1913 (künftig: WUB), Bd. 8, Nr. 3370. Als erweitertes Digitalisat des HStA Stuttgart nutzbar unter www.wubonline.de (05. 06. 2013).

22 Jürgen Sydow, Adlige Stadtgründer in Südwestdeutschland, in: Südwestdeutsche Städte im Zeitalter der Staufer, hg. von Erich Maschke/Jürgen Sydow (Stadt in der Geschichte, Bd.6), Sigmaringen 1980, S.184-192, hier S. 188. Vgl. auch Ernst (wie Anm. 7) S. 124: „Die Erbauung einer Stadt war ein Vorgang innerhalb des eigenen Gebiets, über den man nicht viel zu urkunden brauchte."

${ }^{23}$ Walter Ziegler, Heiningen zur Zeit König Rudolfs von Habsburg (1273-1291), in: 
doch muss dies im Umkehrschluss nicht zwingend bedeuten, dass auch Heiningen zur Stadt aufgestiegen war. Dies beweisen die beiden fast gleichlautenden Stadtrechtsurkunden für Bietigheim und Laichingen von 1364, deren Inhalt nur im Falle Bietigheims zum Tragen kam, während Laichingen ein Dorf blieb ${ }^{24}$. Daneben gilt zu beachten, dass Heiningen in erster Linie Marktrechte zugesprochen wurden, wohingegen andere Bestimmungen, z.B. zur Gerichtsbarkeit, nicht explizit erwähnt werden. Die Privilegierung alleine reicht also nicht aus, um städtischen Anfängen nachzuspüren, und schon gar nicht markiert sie den Anfang als Stadt ${ }^{25}$. Während Heiningen das einzige der hier zu betrachtenden Fallbeispiele darstellt, das über eine königliche Rechtsverleihung und damit über eine etwas konkretere zeitliche Einordnung seiner urbanen Entwicklung verfügt, können bei allen anderen Orten nur Überlieferungszufälle weiterhelfen. Bei diesen handelt es sich um die Erstnennung als Stadt und damit um den Terminus ante quem für die Einschätzung der Stadtwerdungsphase. Gönningen z.B. wird erstmals anlässlich eines Kaufgeschäfts zwischen dem Kloster Zwiefalten und den Grafen von Zollern im Jahr 1287 als Stadt erwähnt (in der Stat ze Ginningen), woraus man sehr grob schloss, dass Gönningen um die Mitte oder in der zweiten Hälfte des 13. Jahrhunderts, zumindest aber vor 1287 zur Stadt erhoben worden sein muss ${ }^{26}$. Gleichermaßen wurde die Stadtgründung Hohenecks, das 1345 zum ersten Mal als oppidum auftaucht, pauschal für einen unbestimmten Zeitraum vor 1345 angesetzt ${ }^{27}$. Allerdings sind solche Datierungen sehr mit Vorsicht zu genießen, wie das Beispiel Gutenberg zeigt. Dieses wird zwar 1370 erstmals als Stadt genannt; bereits 1305 aber erscheint es unter den teckischen Städten, ohne eigens als Stadt tituliert zu sein ${ }^{28}$.

Heiningen. Geschichte und Gegenwart, hg. aus Anlaß der 750-Jahrfeier der Gemeinde Heiningen, Göppingen 1978, S.74-85, hier S. 82. Das Privileg für Sulz ist zu finden in WUB, Bd. 8, Nr. 3384 .

${ }^{24}$ Vgl. Hansmartin Decker-Hauff, Ulm, Helfenstein und Wirtemberg auf der Hohen Alb im 14. Jahrhundert. Gedanken zur Laichinger Stadtgründungsurkunde von 1364, in: Aus Archiv und Bibliothek. Studien aus Ulm und Oberschwaben. Max Huber zum 65. Geburtstag, hg. von Alice Rösslen, Weißenhorn 1969, S. 147-151, hier S. 147.

${ }^{25}$ Die Privilegierung allein reicht aber auch nicht aus, um auf ihre mangelnde Umsetzung zu schließen, wie es Karl Weller tat: Weller (wie Anm. 7) S. 323.

26 WUB, Bd. 9, Nr. 3669. Dieser Hinweis ist Paul Ackermann offensichtlich entgangen, der die Stadtnennung von 1300 für die erste hielt: Paul Ackermann, Gönningens Geschichte und Gegenwart kurzgefaßt, in: Die Gönninger. „Ein Völklein frisch-belebt“. Geschichte und Gegenwart eines Reutlinger Stadtbezirks, hg. von der Stadt Reutlingen, Reutlingen 1992, S. 9-16, hier S. 10. Zur Datierung s. auch LBW, Bd. 7, S. 63.

${ }_{27}$ Herbert Felden, Ortsbuch Hoheneck. Stadtteil von Ludwigsburg (Walter-Ortsbuch, Bd.35), Neckarwestheim 1983, S. 94 und 99; Richard Stein, Chronik von Hoheneck im Oberamt Ludwigsburg, Stuttgart 1921, S. 41.

${ }^{28} \mathrm{Zu} 1370$ s. Irene Gründer, Studien zur Geschichte der Herrschaft Teck (SSWLK, Bd.1), Stuttgart 1963, Nr. 301, S. 170 f. Zu 1305 s. ebd., Nr.113, S. 112. Vgl. auch Rolf GöTZ, Vom Burgweiler zum „Flecken, der Stadtrecht hat“: Die Geschichte Gutenbergs bis zur Mitte des 16. Jahrhunderts, in: Gutenberg. Geschichte einer Gemeinde am Albaufstieg, hg. von der Gemeinde Lenningen, Kirchheim unter Teck 1998, S. 15-55, hier S. 21. 
Die Stadtwerdung fand daher wahrscheinlich im späten 13. Jahrhundert und damit wesentlich früher statt, als es die tatsächliche Erstnennung suggeriert ${ }^{29}$.

Unter ganz anderen Vorzeichen wurde wiederum die Stadtnennung Hohenhaslachs gesehen, das nur ein einziges Mal überhaupt als Stadt Erwähnung findet, nämlich im Jahr 1356, als Graf Heinrich von Vaihingen mit den Grafen von Württemberg die Bereinbarung traf, dem mächtigen Nachbarn nach seinem Tod die Überreste seiner Grafschaft zu überlassen - womit im Wesentlichen das noch verbliebene eselsbergische Erbe mit den beiden Städten, wie die Urkunde sagt, Hohenhaslach und Horrheim gemeint war ${ }^{30}$. Diese einmalige Nennung ließ bezweifeln, ob Hohenhaslach wirklich einen Stadtstatus erreicht hatte. Und tatsächlich erscheint es bis zum Vermächtnis Graf Heinrichs stets als Dorf, wird dann sogar im Jahr des Vermächtnisses in einem Lagerbuch des Klosters Bebenhausen ebenfalls als Dorf bezeichnet und begegnet auch 1372 und 1374 nur als Dorf ${ }^{31}$. Lediglich im Jahr 1364 wird es anlässlich der Abfindung Mechthilds, der Schwester Graf Heinrichs von Vaihingen, die sich ihr Erbe ausbezahlen ließ, einmal als vestin bezeichnet, als befestigter Ort also ${ }^{32}$. Helmut Orth und Otfried Kies folgerten daraus, dass Graf Heinrich von Vaihingen aus unbekannten Gründen einen Etikettenschwindel betrieben habe, als er Hohenhaslach sowie Horrheim in seinem Testament als Städte bezeichnete. Und Mechthild habe durch die Betonung der Wehrhaftigkeit (durch die gemäßigtere Wortwahl vestin) wahrscheinlich etwas mehr Geld herausschlagen können. Es habe sich bei beiden Orten also zwar zweifelsohne um die „Filetstücke“ der noch bestehenden Grafschaft Vaihingen gehandelt, keineswegs jedoch um richtige Städte ${ }^{33}$. Betrachtet man die wenigen Indizien für eine mögliche Stadtwerdung Hohenhaslachs, ergibt sich allerdings kein so klares Bild wie das von Helmut Orth und Otfried Kies angenommene: Das als „Filetstück“ titulierte Hohenhaslach, das bereits zu Zeiten Belreins von Eselsberg gemeinsam mit Horrheim den Mittelpunkt der kleinen Herrschaft markierte,

${ }^{29}$ Vgl. Alois Schneider, Auf geschichtsträchtigem Boden - Beobachtungen zur Siedlungsentwicklung und historischen Topographie von Kirchheim unter Teck, in: Henriettenstift, Lateinschule, Herdstellenverzeichnis und Türkensteuerliste, Schöllkopfkapelle, Siedlungsgeschichte und historische Topografie, Georg Plunckher, hg. vom Stadtarchiv Kirchheim unter Teck (Schriftenreihe des Stadtarchivs Kirchheim unter Teck, Bd.27), Kirchheim unter Teck 2001, S. 133-185, hier S. 149.

30 Württembergische Regesten von 1301 bis 1500, Bd.1/1: Altwürttemberg, hg. von dem K. Haus- und Staatsarchiv in Stuttgart, 3 Bde., Stuttgart 1916 (künftig: WR), Nr. 14112. S. auch die überarbeitete und erweiterte Onlineversion der WR unter www2.landesarchiv-bw. de/ofs21/olf/starbild.php?bestand $=3703$ (05.06. 2013).

31 WUB, Bd. 5, Nr. 1345 (1255); WUB, Bd. 8, Nr. 3242 (1283); WUB, Bd. 8, Nr. 3317 und 3318 (1284); WUB, Bd. 9, Nr.3828 (1289); WR 11789 (1352); Helmut OrTh/Otfried Kies, Hohenhaslach - Stadt oder Dorf?, in: Hohenhaslach. Geschichte und Geschichten aus 1200 Jahren Dorfleben, red. von Peter Schaller/Otfried Kies, Sachsenheim 2000, S. 38-41, hier S. 38 (1356); WR 14219 (1372); WR 14222 (1374).

32 WR 14113.

33 Orth/Kies (wie Anm. 31) S. 40 f. (Zitat auf S. 41). 
dürfte von den Grafen von Vaihingen nach Kräften gefördert worden sein. Es besaß überdies eine Befestigung ${ }^{34}$. Und selbst die noch 1356 und 1372 aufscheinenden Titulierungen als Dorf müssen nicht zwingend zu der Annahme führen, dass Hohenhaslach niemals eine Stadt war. Begriffe wie villa, municipium, oppidum oder civitas wurden nicht einheitlich gebraucht und bedeuteten folglich nicht immer dasselbe. Zudem ist eine Mischung unterschiedlichster Siedlungsbezeichnungen nicht selten bei Stadtwerdungsprozessen. Horb am Neckar beispielsweise wird 1261 als civitas tituliert, in den Jahren 1282 und 1283 jedoch auch zweimal als villa. Gleiches gilt für Waiblingen, das noch 1291 als villa erscheint, und Stuttgart, das 1282 und sogar noch 1301 als villa bezeichnet wird ${ }^{35}$. Rainer Loose erklärt diese Vermischung der Begrifflichkeiten damit, dass die zeitgenössischen Schreiber noch keine Vorstellung von städtischen Siedlungen besessen hätten und für sie Dorf und Stadt noch kaum unterscheidbar gewesen seien ${ }^{36}$. Bezieht man nun noch Horrheim in die Betrachtung ein, dem Helmut Orth und Otfried Kies allein aufgrund der Tatsache, dass es in den Quellen meist gemeinsam mit Hohenhaslach erscheint, ebenfalls pauschal jegliche städtische Entwicklung aberkannt haben, entstehen sogar deutliche Zweifel an ihrer These. Horrheim wird nämlich, außer den beiden gemeinsamen Nennungen mit Hohenhaslach in den Jahren 1356 und 1364, bereits 1304 als Stadt erwähnt und dann noch zweimal in den Jahren 1384 und $1393^{37}$. Schon 1286 sind mit dem Schultheißen und 6 Richtern Verwaltungsorgane belegt, für das Jahr 1304 ist eine Befestigung des Ortes bezeugt und im Jahr 1329 trat Graf Johannes von Vaihingen als Kirchenrektor in Horrheim auf, was darauf hinweist, dass der Ort innerhalb der Kirchenstruktur eine zumindest kurzzeitige Aufwertung erfuhr ${ }^{38}$. Für Horrheim verdichten sich die Anzeichen für einen Stadtwerdungsprozess also in hohem Maße. Berücksichtigt man nun, dass sowohl Horrheim als auch Hohenhaslach offensichtlich die hervorgehobenen Orte der zusammengeschrumpften Grafschaft Vaihingen waren, stellt sich die berechtigte Frage, warum man einen beginnenden oder möglicherweise sogar abgeschlossenen Stadtwerdungsvorgang nicht auch für Hohenhaslach annehmen sollte. Sicher beweisen lässt sich weder der Stadtstatus noch dessen Mangel.

${ }^{34}$ Ebd. (wie Anm. 31) S. 40 f.

35 Zu Horb s. WUB, Bd.6, Nr.1612 (1261); WUB, Bd. 8, Nr. 3127 (1282); WUB, Bd. 8, Nr. 3223 (1283). Zu Waiblingen s. WUB, Bd. 9, Nr. 4083. Zu Stuttgart s. WUB, Bd. 8, Nr. 3163 (1282); Urkundenbuch der Stadt Stuttgart, bearb. von Adolf RAPP (WGQu, Bd.13), Stuttgart 1912, Nr. 32, S. 11 (1301). Zur Terminologie im Allgemeinen s. Jürgen Sydow, Stadtbezeichnungen in Württemberg bis 1300, in: Festschrift für Berent Schwineköper zu seinem 70. Geburtstag, hg. von Helmut Maurer, Sigmaringen 1982, S. 237-248, v. a. S. 241-248. S. 74 .

${ }^{36}$ Rainer Loose, Oberndorfs städtische Anfänge, in: ZWLG 62 (2003) S. 73-84, hier

37 Friedrich Wissmann, 1200 Jahre Horrheim (Kreis Vaihingen/Enz), Ludwigsburg 1972, S. 14.

${ }^{38}$ Ebd. (wie Anm. 37) S. 88. 
Die bisherigen Beispiele machen deutlich, dass die Errichtung einer Stadt weder ein einmaliger Akt war, der wie im Falle Heiningens nur durch eine entsprechende Urkunde besiegelt zu werden brauchte, noch sich an einzelnen Stichdaten festmachen lässt, sondern sich aus verschiedenen, über einen längeren Zeitraum verteilten Vorgängen zusammensetzte, deren Synthese erst die fertige Stadt ins Leben rief. Zu diesen Vorgängen zählten neben der grundsätzlichen Siedlungsanlage Veränderungen in fortifikatorischer Hinsicht durch Befestigungen, in wirtschaftlicher Hinsicht durch die Errichtung eines Marktes, in rechtlicher Hinsicht durch die Ausformung gewisser, an den Ort geknüpfter Privilegien und in sozialer Hinsicht durch die Entwicklung zur Bürgergemeinde sowie der aus all diesen Prozessen resultierende Gewinn zentraler Funktionen, die auf das Umland abstrahlten ${ }^{39}$.

Bei Heiningen zum Beispiel ist für das Jahr 1284 nicht nur das Marktrecht, sondern auch die Befestigung des Ortes belegt - immerhin wird es in der Privilegienverleihung Rudolfs von Habsburg als oppidum bezeichnet. Bereits fünf Jahre zuvor wird der Ministeriale Eberhard von Lotenberg erwähnt, ein Dienstmann der Herzöge von Teck, der sich also nach deren Siedlung Lotenberg benannte oder vielleicht dort ansässig war. Der gleiche Eberhard taucht aber im Jahr 1286 als minister de Huningen auf. Offensichtlich hatte er sich also umbenannt oder sogar seinen Sitz nach Heiningen verlegt, das nach der königlichen Urkunde und im Zuge seiner Stadtwerdung eine Aufwertung erfahren hatte ${ }^{40}$. Als weiteres Indiz lässt sich noch die Erwähnung des Dekans Albert von Heiningen im Jahr 1275 anführen, der zudem eine Pfründe in Faurndau besaß. Heribert Hummel hat dieses zusätzliche Kanonikat auf den Einfluss der Herzöge von Teck zurückgeführt, die Heiningen wahrscheinlich auch in kirchlicher Hinsicht stärken wollten ${ }^{41}$.

Für Hoheneck ist neben seiner Ummauerung auch eine privilegierte Rechtsstellung seiner Bürger nachgewiesen, die keinen Leibzins zahlen mussten und auch zu Forst- und Jagdfronen nur eingeschränkt herangezogen wurden. Das Kloster Bebenhausen erhielt überdies 1291 die Erlaubnis, einen Pfleghof im Ort errichten zu dürfen, was möglicherweise bereits auf den beginnenden Aufschwung der Siedlung hinweist ${ }^{42}$.

Die Stadtwerdung war also ein vielteiliger und bisweilen langwieriger Prozess, dessen Vollendung mangels Quellen kaum terminiert werden kann. Gewährt uns bei Heiningen und Hoheneck die sehr kleine Zahl an Belegen, die als Indikatoren für Stadtwerdungsvorgänge gelten dürfen, aber zumindest noch einen geringfügigen Einblick in die Transformation der Siedlung, so ist dies in anderen Fällen so

39 Vgl. Engel (wie Anm.6) S. 35 f.; Isenmann (wie Anm.5) S.24f.; Schmieder (wie Anm. 14) S. 3 und 23.

40 Hans Bauer, Streifzug durch die Geschichte Heiningens, in: Heiningen (wie Anm. 23) S. 9-73, hier S. 19.

${ }^{41}$ Heribert Hummel, Die Pfarrei im Mittelalter, in: Heiningen (wie Anm. 23) S. 181-191, hier S. 182-184.

${ }^{42}$ Stein (wie Anm. 27) S. 41 und $129 \mathrm{f}$. 
gut wie gar nicht mehr möglich. Die einzelnen Faktoren der urbanen Entwicklung Gutenbergs etwa lassen sich fast nur aus der Rückschau rekonstruieren. So weisen erst die Erwähnung eines Grabenmüllers aus dem Jahr 1502 und ein entsprechender Lagerbucheintrag von 1513 auf die Befestigungsanlagen des Ortes hin ${ }^{43}$. Ein Lagerbuch von 1561 gibt Aufschluss darüber, dass die Einwohner Gutenbergs, des Fleckhens, das Stetrecht hat, seit alters keine Leibeigenschaftsabgaben zu zahlen hatten $^{44}$. Und der Chronist Daniel Wolleber erwähnt 1590, dass Gutenberg, wie die Privilegia Und alte gemeur gnugsam anzaigen, Ein Stättlin gewesen sei ${ }^{45}$.

Hier stoßen wir auf ein Charakteristikum der „Statuswüstungen“, deren Dasein als Stadt häufig so kurz war, dass Nachweise für die Stadtwerdung nur selten einen schriftlichen Niedergang fanden, und dass sich manches nur aus der bereits fernen Erinnerung der älteren Chronistik oder den akribisch angelegten, frühere Zustände konservierenden Lagerbüchern des 16. Jahrhunderts nachvollziehen lässt. Dass die sehr vereinzelten Nachrichten zur Stadtwerdung infolgedessen einer erheblichen Interpretation und Kombination bedürfen, war bei Hohenhaslach zu sehen, dessen Status sich nicht sicher beweisen lässt, und auch der Stadtstatus des bislang noch nicht erwähnten Ochsenburg gilt in Ermangelung einschlägiger Stadtwerdungsbelege als fraglich ${ }^{46}$. Wie groß die entstehenden Interpretationsspielräume tatsächlich sein können, tritt jedoch in keinem Fall so klar zu Tage wie bei Neustadt, das uns zu unserer nächsten Frage nach den Stadtgründern und ihren Motiven führt.

\section{Stadtgründer und ihre Motive}

Das späte 13. Jahrhundert war für Württemberg eine Zeit zahlreicher kriegerischer Auseinandersetzungen. Nach der Königswahl Rudolfs von Habsburg 1273, der es sich zum Ziel gesetzt hatte, widerrechtlich in Besitz genommenes Reichsgut für die Krone zurückzugewinnen, kam es zu wiederholten Zusammenstößen zwischen Graf Albrecht von Hohenberg, Schwager Rudolfs und zugleich Landvogt in Schwaben, und Graf Eberhard I. von Württemberg, der die Rückgabe der geforderten Güter verweigerte. Auch unter Rudolfs Nachfolger Adolf von Nassau verbesserte sich die Situation nicht: Eberhard I. verweigerte den Treueid, wahrscheinlich weil ihm Heinrich von Isenburg als neuer Landvogt von Schwaben vorgezogen worden war, und musste abermals Verwüstungen und Burgbesetzungen in seinem Herrschaftsgebiet hinnehmen. Dies führte dazu, dass er sich 1298 auf die Seite des Thronanwärters Albrecht von Habsburg begab und ihm zusagte, ihn bei der Erlan-

${ }^{43}$ Karl Mayer, Chronik des ehemaligen Städtleins Gutenberg am Fuß der schwäbischen Alb, Kirchheim unter Teck 1948, S. $11 \mathrm{f}$.

${ }^{44}$ Götz (wie Anm. 28) S. 25 f.

45 Zitiert nach ebd., S. 25.

46 Z.B. bei Schaав (wie Anm. 9) S. 225. 
gung der Königswürde zu unterstützen. Im Zuge dieser Vereinbarung war plötzlich erstmals von dem stättelein daz Neu Weibelingen die Rede, das Albrecht nach seiner Königswahl zusammen mit der Burg Rems an Württemberg zu geben versprach, und bei dem es sich um den heutigen Waiblinger Stadtteil Neustadt handelt $^{47}$. Doch was hatte es damit auf sich, wer hatte es gegründet und warum wollte Eberhard I. es in seinen Besitz bringen?

Wie sehr Neustadt, das so überraschend zu einem Vertragsgegenstand des künftigen Königs Albrecht von Habsburg und des Grafen Eberhard I. von Württemberg wurde, die Phantasie von Historikern und Heimatforschern anregte, zeigt sich darin, dass es allein fünf unterschiedliche Versionen zu seiner Gründungsgeschichte gibt und der Ort gar zum siebenjährigen „Zentralpunkt“ deutscher Geschichte stilisiert wurde ${ }^{48}$. Freilich können im Rahmen dieses Beitrags nicht alle Feinheiten der Neustädter Geschichte besprochen werden, jedoch wollen wir einen Blick auf die verschiedenen Gründungsfassungen und die dahinter stehenden Motive werfen. So soll nach einer Sage die Stadt Waiblingen um das Jahr 1000 bereits so groß geworden sein, dass die Einwohner eine neue Stadt an die alte Ringmauer bauten $^{49}$. Da es sich dabei lediglich um eine sagenhafte Verklärung Waiblingens handelt, die allen historischen Fakten widerspricht, lassen wir diese erste Version getrost bei Seite und wenden uns der zweiten zu: Nach ihr soll Neustadt um 1260/70 als Neuanlage Waiblingens geplant gewesen sein, weil es sich mit seiner Lage auf einem Vorsprung in militärischer Hinsicht wesentlich besser eignete. In Folge der Kriegswirren sei diese Anlage dann an das feindliche Lager gekommen, um erst 1298 wieder an Württemberg zurückzugelangen ${ }^{50}$. Auch diese Fassung klingt wenig plausibel, da Waiblingen erst kurz zuvor um 1250 zur Stadt erhoben worden war, über eine günstige Verkehrslage an der Remstalstraße nach Nördlingen und Donauwörth verfügte und ein beliebter Aufenthaltsort der württembergischen Grafen war ${ }^{51}$.

Nach einer dritten Version soll Graf Eberhard I. selbst in den Jahren 1291/92 das neue Waiblingen als Ersatz für das alte gegründet haben, weil letzteres während

47 WUB, Bd.11, Nr. 5128. Zu den geschilderten Ereignissen vgl. Dieter Mertens, Württemberg, in: Handbuch der baden-württembergischen Geschichte, Bd. 2: Die Territorien im Alten Reich, hg. von Meinrad SchaAb/Hansmartin Schwarzmaier, Stuttgart 1995, S. 1-163, hier S.23-28; Ellen Widder, Die erste Stadt Württembergs? Waiblingen im Spätmittelalter, in: Waiblingen. Eine Stadtgeschichte, hg. von Sönke Lorenz (Gemeinde im Wandel, Bd.13/2), Filderstadt 2003, S.81-147, hier S.118f.; Werner Haupt, Das „Niuwe Waibelingen“. Zankapfel deutscher Geschichte, in: Waiblingen in Vergangenheit und Gegenwart 9 (1987) S. 123-134, hier S. 123, 125 und 127.

48 Ebd., S. 132.

49 Emil Dietz, Chronik Neustadt (Kreis Waiblingen), Bd.1: 1289-1918, Neustadt 1969, S. $2 \mathrm{f}$.

50 Ernst (wie Anm. 7) S. 130; Dietz (wie Anm. 49) S. 3.

51 Widder (wie Anm. 47) S. 117 und 146; Gerhard Fritz, Waiblingen und Umgebung im 12. und 13. Jahrhundert. Studien zur Waiblinger Stadtgeschichte, in: Waiblingen in Vergangenheit und Gegenwart 11 (1990) S. 21-49, hier S. 44. 
der Auseinandersetzungen mit Albrecht von Hohenberg mehrfach zerstört worden sei und der Württemberger die obdachlosen Waiblinger Bürger habe unterbringen wollen ${ }^{52}$. Über das tatsächliche Ausmaß der Zerstörungen Waiblingens ist jedoch nichts bekannt, zumal Graf Eberhard I. 1293 ein Fest in Waiblingen feierte, wahrscheinlich als Demonstration seines ungebrochenen Willens, und bereits 1304 das Schmidener und das Fellbacher Tor fertiggestellt waren ${ }^{53}$ - eine Mühe, die man bei einer Siedlungsverlegung kaum hätte auf sich nehmen müssen. Zudem leuchtet es wenig ein, warum der Württemberger seine Stadt aufgrund von Kriegszerstörungen hätte verlegen sollen, anstatt sie schnell wiederaufzubauen, wie es wahrscheinlich auch geschehen ist.

Nach einer leicht abweichenden Variante soll Eberhard I. Neustadt zum gleichen Zeitpunkt gegründet haben, nun aber, um gewissermaßen ein Bollwerk gegen die bereits erwähnte, von königlicher Seite errichtete Burg anzulegen. Als wichtiges Argument gilt dabei, dass die Ringmauer Neustadts sich gegen die Burg gerichtet habe und die jeweiligen Gründer folglich Kontrahenten gewesen sein müssen ${ }^{54}$. Joachim Peterke erwähnt jedoch in seinem Abriss zur mittelalterlichen Geschichte Neustadts, dass es das Stadtbauamt Waiblingen anlässlich einer Ortsbegehung durchaus für denkbar gehalten habe, dass Neustadt neben den beiden bekannten Stadttoren noch ein drittes in Richtung der Burg besessen habe - ein Hinweis, den der Autor nicht gelten lässt, obwohl schon Martin Crusius von drei Neustadter Toren berichtet hatte ${ }^{55}$.

Wirklich überzeugt hat bislang noch keine der verschiedenen Fassungen, so dass schließlich eine letzte zu betrachten bleibt, die auch am wahrscheinlichsten ist. Nach dieser wurde Neustadt um 1289 von Albrecht von Hohenberg, dem Gegenspieler Graf Eberhards I., als Konkurrenz zu Waiblingen und königlicher Vorposten angelegt, als „Trutz-Waiblingen“ und „Pfahl im Fleische“, um sowohl Waiblingen selbst als auch Stuttgart besser kontrollieren zu können ${ }^{56}$. Der Name

52 Werner Haupt, Die Anfänge der Ortschaft Waiblingen-Neustadt, in: Waiblingen in Vergangenheit und Gegenwart 6 (1980) S. 94-108, hier S. 100. Werner Haupt interpretiert lediglich die als Burg gedeutete nova civitas als gegnerische Anlage, während Neuwaiblingen eine württembergische Gründung sei. S. auch Ders. (wie Anm. 47) S. 126 und 130.

${ }^{53}$ Vgl. Karl Eisele, Aus der Geschichte der Stadt Waiblingen, Waiblingen 1896, S. 9; Fritz (wie Anm.51) S.44; Karl Stenzel, Waiblingen in der deutschen Geschichte, in: WVjH 38 (1932) S. 164-212, hier S. 208.

54 Joachim Peter ke, Neustadt im Spätmittelalter, in: Neustadt. Die ungewöhnliche Geschichte eines schwäbischen Dorfes - heute Stadtteil von Waiblingen im Remstal, hg. von Joachim Peterke, Neustadt 2009, S. 21-57, hier S. 32. Vgl. auch Haupt (wie Anm. 52) S. 98.

55 Joachim Peterke bemerkt dazu: „Crusius wird allerdings wohl nie in Neustadt gewesen sein und daher weder Orts- noch vollständige Quellenkenntnis gehabt haben." (WILD [wie Anm. 54] S. 36). Vgl. hingegen LBW, Bd.3, S. 561, wo man der Darstellung von Crusius folgt.

56 So bei Eggert (wie Anm. 9) S. 161; Weller (wie Anm. 7) S. 336; Stenzel (wie Anm. 53) S. 208 (hier das erste Zitat); Widder (wie Anm. 47) S. 119 (hier das zweite Zitat). 
Neuwaiblingen wurde dabei als Drohung gegen Württemberg interpretiert ${ }^{57}$. Er könnte jedoch auch durch ehemalige Waiblinger entstanden sein, die sich hier ansiedelten, weil sie unter Graf Albrecht von Hohenberg oder gar dem König mehr Schutz oder vielleicht einfach nur bessere Lebensbedingungen erwarteten. Dass Graf Eberhard I. diesen Ort 1298 für sich forderte, leuchtet unmittelbar ein. Nach den schweren Auseinandersetzungen mit dem König konnte er weder dulden, dass sich in solcher Nähe eine gegnerische Stadtgründung befand, noch dass hier eine direkte Konkurrenz zum württembergischen Waiblingen entstand. Ähnlich handelten die Württemberger auch in anderen Fällen, etwa 1334, als man die Stadt Grötzingen von den Herren von Bernhausen erwarb, um die Entwicklung des nahen, selbst gegründeten Nürtingen nicht zu gefährden ${ }^{58}$. Dass die Württemberger allerdings vorhatten, Neustadt zu zerstören, und König Albrecht daher urkundlich verfügte, Neustadt müsse bestehen bleiben, beruht auf einer Fehlinterpretation der entsprechenden Urkundenstelle ${ }^{59}$.

Wir sehen also, dass mit einer Stadtgründung ganz unterschiedliche Motive verbunden sein konnten, die ihrerseits vom Gründer, dem Gründungszeitpunkt und den räumlichen Voraussetzungen abhängig waren. Im Vergleich zeigt sich, dass es sich bei den hier untersuchten Städten durchweg um späte Stadtgründungen aus der zweiten Hälfte des 13. Jahrhunderts oder der ersten Hälfte des 14. Jahrhunderts handelte und dass alle Gründer kleine, nur lokal agierende Herren waren, wie die Herren von Stöffeln (Gönningen), Johann II. von Rechberg (Hoheneck) und die Herren von Enzberg (Ochsenburg), bzw. ehemals glanzvollen, nun aber zunehmend verschuldeten Geschlechtern angehörten, wie den Herzögen von Teck (Gutenberg und Heiningen) oder den Grafen von Vaihingen (Hohenhaslach und Horrheim $)^{60}$. Diese Parameter übten einen erheblichen Einfluss auf die städtische Entfaltung aus, denn späte Stadtgründungen trafen im dichten Städtenetz Südwestdeutschlands auf zahlreiche ältere und etablierte Konkurrenz, verfügten häufig über nur defizitäre Lagen und entwickelten sich aufgrund dieser Hemmnisse in der Regel nur noch zu Kleinstädten. Die Motive ihrer Gründer, die meist über nur

57 Ebd., S. 119.

58 WR 11411. Vgl. z. B. Heimatbuch des Kreises Nürtingen, hg. von Hans SCHwenkel, 2 Bde., Nürtingen 1953, Bd.2, S.263. Weitere Beispiele bei Widder (wie Anm. 47) S. 119.

59 WUB, Bd.11, Nr.5188. Joachim Peterke bezieht die Wendung daz staete belibe auf Neustadt; tatsächlich ist sie jedoch Teil des Beurkundungsbefehls: PETERKe (wie Anm. 54) S. 42 und 50 .

60 Hansmartin Decker-Hauff ging davon aus, dass um die Mitte des 13. Jahrhunderts noch die Herren von Eselsberg Horrheim und Hohenhaslach zu Städten erhoben hätten: Hansmartin Decker-Hauff, Wer gründete Kloster Rechentshofen?, in: Hie gut Württemberg 1 (1950) S. 86 f., hier S. 87. Und Gerhard Fouquet vermutet in den Grafen von Vaihingen oder den Grafen von Württemberg die Stadtgründer Ochsenburgs: Gerhard FouQuet, Stadt, Herrschaft und Territorium - Ritterschaftliche Kleinstädte Südwestdeutschlands an der Wende vom Mittelalter zur Neuzeit, in: ZGO 141 (1993) S. 70-120, hier S. 93. 
eng umgrenzte Herrschaften geboten, waren dabei überwiegend territorialpolitischer Natur.

Heiningen beispielsweise lag von den eigentlichen Kerngebieten der Herzöge von Teck im Kirchheimer Raum weit entfernt und sah sich von Besitzungen der Grafen von Württemberg und der Grafen von Helfenstein umgeben ${ }^{61}$. Dies machte den Ort zu einem geeigneten teckischen Vorposten, dessen Befestigung ebenso wichtig war wie seine wirtschaftliche Förderung durch ein königliches Privileg. In den Kriegswirren des späten 13. Jahrhunderts fand Herzog Konrad von Teck, der auf der Seite Rudolfs von Habsburg gegen Württemberg kämpfte, jedoch keine Zeit für den Ausbau Heiningens, und nach dem Krieg setzte bereits der wirtschaftliche Niedergang der Herzöge von Teck ein. Eine vielleicht geplante Neuanlage Heiningens unterblieb daher ebenso wie eine feste Ummauerung; Heiningen war weiterhin nur mit Gräben, Wällen und Hecken befestigt und behielt die dörfliche Siedlungsfläche auch künftig bei ${ }^{62}$.

Im Falle Gutenbergs vermutete man, dass die Herzöge von Teck durch dessen Gründung den Verlust der Stadt Kirchheim kompensieren wollten, von der sie bereits zu Beginn des 14. Jahrhunderts die eine Hälfte an die Habsburger verkauft und 1359 die andere Hälfte an Württemberg verpfändet hatten ${ }^{63}$. Doch Gutenberg dürfte, wie gezeigt, schon im späten 13. Jahrhundert Stadt geworden sein und reihte sich damit in die zahlreichen teckischen Stadtgründungen ein. Die Grafen von Vaihingen schließlich versprachen sich von Hohenhaslach und Horrheim ebenso eine Stärkung ihrer Herrschaftsposition wie die Herren von Stöffeln von ihrer Gründung Gönningen, die, unterhalb ihrer Burg liegend, durch Mauern, Gräben und den Flussverlauf der Wiesaz als gut befestigt gelten konnte ${ }^{64}$.

Gegenüber den territorialpolitischen Zielsetzungen gerieten wirtschaftliche Erfordernisse meist ins Hintertreffen. Ohnehin waren die städtischen Möglichkeiten in dieser Hinsicht bereits durch die Verkehrslage der Dorfsiedlung determiniert, die bei einer neuen, planmäßigen Stadtanlage zwar geringfügig korrigiert, aber nicht entscheidend beeinflusst werden konnte. Die abseitige Lage Heiningens wurde bereits thematisiert; auch lag es in unmittelbarer Nachbarschaft zum älteren und größeren Göppingen, was sich auf die weitere städtische Entwicklung negativ

${ }^{61}$ Ziegler (wie Anm. 23) S. 76; Gründer (wie Anm. 28) S. 11; Bauer (wie Anm. 40) S. 13 und 15.

${ }^{62}$ Ebd., S. 27; Ziegler (wie Anm. 23) S. 79f.; Hartmut Schäfer, Die Ortsbefestigung von Heiningen, in: Archäologische Ausgrabungen in Baden-Württemberg 3 (1983) S.233f.

${ }^{63}$ Vgl. Heimatbuch Nürtingen (wie Anm. 58) Bd. 1, S. 308. Zur Veräußerung Kirchheims s. Rolf Gӧтz, Geschichte Kirchheims von der ersten urkundlichen Nennung im Jahre 960 bis zur Mitte des 16. Jahrhunderts, in: Kirchheim unter Teck. Marktort, Amtsstadt, Mittelzentrum, hg. von Rainer Kilian, Kirchheim unter Teck 2006, S. 97-273, hier S. 143 und 151.

64 Wilhelm Kinkelin spricht im Falle Gönningens gar von einer „Doppelburg“: Wilhelm Kinkelin, Heimatbuch Gönningen aus Anlaß der 860-Jahrfeier, Gönningen 1952, S. 27. Zu Hohenhaslach und Horrheim s. Erich BuHL, Hohenhaslach im Zeitlauf der Geschichte, Hohenhaslach 1970, S. 38. 
auswirken musste. Hohenhaslach befand sich zwar ebenso wie Horrheim am Handelsweg in Richtung Heilbronn und Pforzheim, verfügte aber über zu wenig Hinterland und war zudem durch seine Lage auf einem steil abfallenden Vorsprung wirtschaftlich im Nachteil ${ }^{65}$. Horrheim hingegen lag in der Ebene neben dem Übergang über die Metter - Voraussetzungen, die möglicherweise zu der beschriebenen schnelleren urbanen Entwicklung beitrugen -, doch auch hier gab es kein ausreichendes Hinterland ${ }^{66}$. Über Gutenberg ließ sich zwar der Albaufstieg in Richtung Esslingen, Kirchheim und Ulm erreichen, jedoch eignete sich das enge und steile Lautertal für landwirtschaftliche Belange denkbar schlecht; dafür besaß die junge Stadt aber eine größere Markung mit finanziell ergiebigen Mühlen, auf die sie ihre Hoffnungen setzen konnte ${ }^{67}$. In Gönningen schließlich kreuzten sich immerhin alte Talwege, die allerdings nicht durch die Stadt selbst führten, so dass sich selbige gezwungen sah, ihren Markt vor das Stadttor an die Weggabelung zu verlegen. Wie bei Gutenberg waren auch hier die landwirtschaftlichen Voraussetzungen schlecht, da Gönningen von Berghängen umgeben war ${ }^{68}$.

Unsere Fallbeispiele wurden von ihren Stadtgründern also zwar mit bestimmten strategischen Funktionen innerhalb ihrer kleinen Herrschaftsgebiete bedacht, das ökonomische Potential spielte dabei aber nur eine untergeordnete Rolle ${ }^{69}$. Dies musste sich entsprechend auf die urbane Entwicklung auswirken, zumal die Stadtherren sich nicht selten selbst in einer prekären finanziellen Situation befanden, wie die Herzöge von Teck oder die Grafen von Vaihingen, und ihre Städte kaum fördern konnten. Wir wollen uns daher, nachdem nun die Grundlagen und Voraussetzungen der Städteauswahl skizziert wurden, als nächstes den weiteren städtischen Entwicklungsphasen zuwenden und hier vor allem die Bedeutung des Übergangs an Württemberg in den Blick nehmen.

${ }^{65}$ Vgl. Manfred Schecк, Die Gründung der Stadt Vaihingen und ihre Entwicklung im 13. Jahrhundert, in: 750 Jahre Stadt Vaihingen. Aufsätze zur Entwicklung der Stadt, hg. von Ernst Eberhard Sснмідт (Schriftenreihe der Stadt Vaihingen an der Enz, Bd. 6), Vaihingen 1989, S. 16-55, hier S. 51; Decker-Hauff (wie Anm. 60) S. 87; Beschreibung des Oberamts Vaihingen, hg. von dem königlichen statistisch-topographischen Bureau, Stuttgart 1856, ND Magstadt 1974 (künftig: OAB Vaihingen), S. 163.

${ }_{66}^{6}$ Decker-Hauff (wie Anm.60) S. 87; Wissmann (wie Anm. 37) S. 14.

67 GöTz (wie Anm. 28) S.15; Heimatbuch Nürtingen (wie Anm. 58) Bd.1, S. 308; Beschreibung des Oberamts Kirchheim, hg. von dem königlich statistisch-topographischen Bureau, Stuttgart/Tübingen 1842, ND Magstadt 1962, S.186.

${ }^{68}$ Kinkelin (wie Anm. 64) S. 27; Beschreibung des Oberamts Tübingen, hg. von dem königlichen statistisch-topographischen Bureau, Stuttgart 1867, ND Magstadt 1970 (künftig: OAB Tübingen), S. 380 .

69 Ähnliche Beobachtungen finden sich bei Ammann, Waadt (wie Anm. 8) S. 93; Weller (wie Anm. 7) S. 352; Flückiger-Seiler (wie Anm. 8) S. 175. 


\section{Städtische Entwicklung in vorwürttembergischer und württembergischer Zeit}

Waren bereits die Hinweise zur Stadtwerdung unserer Untersuchungsbeispiele eher rar gesät, so gilt dies gleichermaßen für die kurze Phase urbaner Entwicklung in vorwürttembergischer Zeit. Dies ist wenig verwunderlich, da alle Orte bereits zu einem sehr frühen Zeitpunkt ihrer städtischen Geschichte an Württemberg kamen. Nichtsdestoweniger sollen einige wenige Beispiele ein ungefähres Bild von ihrer Bedeutung vermitteln, um später einen deutlicheren Vergleich mit den Zuständen unter württembergischer Herrschaft ziehen zu können.

So fällt auf, dass manche Städte zunehmend zentrale Funktionen erhielten. Am deutlichsten wird dies bei Gutenberg erkennbar, das jedoch von allen Beispielen auch am längsten im Besitz seiner Stadtgründer blieb. Gutenberg wurde wahrscheinlich zum Mittelpunkt eines teckischen Verwaltungs- und Gerichtsbezirks, wobei seine Zentralität durch die Anwesenheit der Herzöge, die sich nach der Verpfändung ihrer halben Herrschaft Teck hierher zurückgezogen hatten, noch gesteigert worden sein dürfte ${ }^{70}$. Dass sich auf der Burg Hohengutenberg aber auch das Archiv der Herzöge befunden haben soll, ist nicht richtig und geht auf einen Überlieferungsirrtum zurück ${ }^{71}$.

Neben Gutenberg war das teckische Heiningen ebenfalls Mittelpunkt eines Amtsbezirks, während Horrheim und wahrscheinlich auch Hohenhaslach Gerichtsbezirke bildeten ${ }^{72}$. Die hohe Gerichtsbarkeit lässt sich außerdem bei Gönningen, Heiningen und Hoheneck über die Flurnamen „Galgenbühl“, „Am Galgenacker" und „Schelmental“ nachweisen, die alle Nachklänge früherer Richtstätten darstellen ${ }^{73}$. Dass ein Verkaufsgeschäft zwischen dem Kloster Zwiefalten und den Grafen von Zollern gerade in Gönningen zum Abschluss kam, kann auf eine relative Wichtigkeit der Stadt hindeuten; zumindest aber schien Gönningen für beide Parteien reisetechnisch günstig gelegen $\mathrm{zu} \operatorname{sein}^{74}$.

In wirtschaftlicher Hinsicht verfügten die Beispiele, wie oben gezeigt, über keine guten Voraussetzungen; dennoch lassen sich auch hier Anhaltspunkte erkennen. Gönningen besaß den besagten Markt, der außerhalb der Stadt an der Wegkreuzung stattfinden musste; daneben ist für Gutenberg ein Jahrmarkt nachgewiesen und auch Horrheim hatte wahrscheinlich einen Wochenmarkt ${ }^{75}$. Inwiefern Heinin-

70 Heimatbuch Nürtingen (wie Anm.58) Bd.2, S.1011; Gründer (wie Anm. 28) S.34; Götz (wie Anm. 28) S. 20; Mayer (wie Anm. 43) S. 12 f.

71 Vgl. GöTz (wie Anm. 28) S. 25.

72 Gründer (wie Anm. 28) S. 9; Wissmann (wie Anm. 37) S. 93.

$73 \mathrm{Zu}$ Gönningen s. Kinkelin (wie Anm.64) S.27. Zu Heiningen s. Ziegler (wie Anm. 23) S. 81. Zu Hoheneck s. Stein (wie Anm. 27) S. 132.

74 Vgl. Heinz Alfred Gemeinhardt, Die früheste schriftliche Erwähnung des Dorfes Gönningen, in: Die Gönninger (wie Anm. 26) S. 17-24, hier S. 23.

$75 \mathrm{Zu}$ Gutenberg s. Götz (wie Anm. 28) S. 22. Zu Horrheim s. Wissmann (wie Anm. 37) S. 109. 
gen von seinem königlichen Marktprivileg Gebrauch machte, ist nicht bekannt. Johann II. von Rechberg erhielt darüber hinaus noch von Ludwig dem Bayern das Recht, bei Hoheneck eine Mühle zu errichten ${ }^{76}$.

Mit dem Übergang an die Grafschaft Württemberg änderten sich die Rahmenbedingungen für die Beispielstädte grundlegend. Die Grafen verfolgten seit der Mitte des 13. Jahrhunderts einen umfassenden Expansionskurs, der mit dem Kauf der Grafschaft Urach zunächst zur Schwäbischen Alb führte, sich bald aber auch bis ins Zabergäu im Norden und bis in den Schwarzwald im Westen orientierte. Begünstigt durch hohe finanzielle Mittel, kauften sie zahlreiche Herrschaften und Städte und erreichten auf diese Weise ein nahezu geschlossenes Herrschaftsgebiet $^{77}$. Unter dem Stichwort der territorialen, flächendeckenden Expansion sind auch die Erwerbungen der Städte Gönningen, Gutenberg, Heiningen, Hoheneck, Hohenhaslach, Horrheim, Neustadt und Ochsenburg einzuordnen. Nach Neustadt, das, wie oben gezeigt, per Vertrag an Württemberg fiel, gelangte als zweite dieser Städte Gönningen an die Grafschaft, dessen Kauf im Jahr 1300 von den Herren von Stöffeln in Verbindung mit der herrschaftlichen Festsetzung auf der mittleren Alb zu sehen ist ${ }^{78}$. Finanzielle Schwierigkeiten der Stadtherren dürften aber ebenso eine Rolle gespielt haben wie bei den Herzögen von Teck, die sich infolge chronischer Geldnöte zu einem regelrechten Ausverkauf ihrer Besitzungen gezwungen sahen. Im Jahr 1321 verpfändeten sie zunächst Boll und Heiningen an Württemberg für 2.000 Pfund Heller, wobei der Einlösungsverzicht Herzog Friedrichs von Teck 1352 erfolgte, und ließen, neben vielen anderen Gütern und Städten, 1383 das Lenninger Tal mit Gutenberg und Owen folgen, das zunächst pfandweise und ab 1387 endgültig an Württemberg überging ${ }^{79}$. Hohenhaslach und Horrheim kamen durch die testamentarische Verfügung des Grafen Heinrich von Vaihingen aus dem Jahr 1356 an Württemberg, der dem mächtigen Nachbarn kurzerhand die Überreste der Grafschaft Vaihingen vermachte und damit einen wichtigen Baustein für die Gebietserweiterung nördlich der Enz lieferte. Wie dieses Vermächtnis zustande kam, ist unbekannt; es zog allerdings eine Entschädigung

76 Vgl. Stein (wie Anm. 27) S. 41.

77 S. z.B. Elmar Blessing, Die territoriale Entwicklung von Württemberg bis 1796 einschließlich der linksrheinischen Besitzungen (Beiwort zur Karte VI,2) (HABW Erläuterungen, Bd. 1), Stuttgart 1972; Peter RüCKeRT, Dynastie, Hof, Territorium. Zur Herrschaftsbildung der Grafen von Württemberg im späteren Mittelalter, in: Das Land am mittleren Neckar zwischen Baden und Württemberg, hg. von Hansmartin Schwarzmaier/Peter RüCKERT (Oberrheinische Studien, Bd. 24), Ostfildern 2005, S. 189-211.

78 WUB, Bd.11, Nr. 5481. Vgl. auch Kinkelin (wie Anm. 64) S. 40.

$79 \mathrm{Zu}$ Heiningen s. WR 8358 und 8370. Zu Gutenberg s. WR 9798 und 9813. Vgl. dazu auch Gründer (wie Anm.28) S. 34 und 41 f.; Götz (wie Anm. 28) S.24f.; Mayer (wie Anm. 43) S.13f. Karl Mayer erwähnt im Zusammenhang mit dem Verkauf Gutenbergs auch den Hinweis des Chronisten Gratianus, dass 1345 ein Erdbeben in Schwaben zehn Burgen, darunter Hohengutenberg, zerstört habe, und vermutet darin eine der Quellen für die erheblichen finanziellen Probleme der Herzöge von Teck (ebd., S. 54). 
für Graf Heinrichs Schwester Mechthild nach sich, die im Jahr 1364 die Summe von 7.500 Pfund Heller von den Württembergern erhielt ${ }^{80}$. Mit dem Vaihinger Besitz ging wahrscheinlich auch die Lehnsherrlichkeit über halb Ochsenburg an die Grafschaft über, von dem noch fast gar nicht die Rede war $^{81}$. Leider ist es bislang kaum Gegenstand wissenschaftlicher Untersuchungen gewesen und blickt noch dazu auf eine recht verworrene mittelalterliche Besitzgeschichte zurück. So gelangte es von den Herren von Magenheim über mehrere Etappen an die Grafen von Vaihingen, die es an Albrecht von Gemmingen, genannt von Enzberg, verpfändeten, ehe es durch das Testament Graf Heinrichs an Württemberg gelangte. Fast zeitgleich folgte ihm die zweite Hälfte Ochsenburgs, die über andere Linien an Württemberg kam ${ }^{82}$. Die Stadtgründung Ochsenburgs dürfte allerdings schon vor 1360 unter besagtem Albrecht von Gemmingen vorgenommen worden sein, ohne dass sich dies mit Sicherheit feststellen ließe. Hoheneck schließlich wurde gleichfalls im Jahr 1360 von Hans von Rechberg, dem Sohn des Stadtgründers, an die Württemberger veräußert und von diesen, nach einem Generalverzicht der erbberechtigten Hacken von Hoheneck, postwendend an selbige verpfändet. Erst 1496/97 wurde es von Württemberg ausgelöst und zählte nun zum festen Bestand des mittlerweile zum Herzogtum aufgestiegenen Territoriums ${ }^{83}$.

Waren die Beispielstädte vor ihrem Übergang an Württemberg bevorzugte Orte innerhalb kleiner Herrschaftsgebiete gewesen, die auf die Bedürfnisse und das Vermögen ihrer früheren Stadtherren zugeschnitten waren und allmählich, wie oben skizziert, rudimentäre zentrale Funktionen entwickelten, stießen sie nun im neuen Herrschaftsverband auf ganz andere Strukturen. In Württemberg kam es schon im laufenden 14. Jahrhundert zur Ämterbildung, einem Verwaltungsaufbau, bei dem jeweils eine Stadt und je nach Region eine unterschiedlich große Zahl an Dörfern zu einem Amtsbezirk zusammengefasst wurden. Der Stadt, die als Mittelpunkt des Amtes zur Amtsstadt aufstieg, fielen dabei zentrale Funktionen in administrativer, wirtschaftlicher und rechtlicher Hinsicht $\mathrm{zu}^{84}$. Aufgrund der dich-

80 WR 14112 (das Testament Graf Heinrichs von Vaihingen); WR 14113 (die Abfindung Mechthilds).

${ }^{81}$ Der Landkreis Heilbronn, hg. vom Landesarchiv Baden-Württemberg (Baden-Württemberg - Das Land in seinen Kreisen), 2 Bde., Ostfildern 2010 (künftig: KB Heilbronn), Bd.2, S. 517.

${ }^{82}$ Zur Besitzgeschichte Ochsenburgs vgl. ebd., Bd.2, S. 517 f.; LBW, Bd. 4, S. 87.; Gerhard Assfahl, Burg und Schloß Ochsenburg und seine Bewohner, in: Zeitschrift des Zabergäuvereins (1984) S. 53-84, hier S. 53; Karl Klunzinger, Geschichte des Zabergäus und des jetzigen Oberamts Brackenheim, Stuttgart 1844, ND Magstadt 1984, S. 109-114 und 229.

${ }^{83}$ Vgl. WR 9577 und 9578; Felden (wie Anm. 27) S. 102.

${ }^{84}$ Zur Amtsentwicklung s. Grube (wie Anm. 12) S. 1-10; Walter Grube, Stadt und Amt in Altwürttemberg, in: Stadt und Umland. Protokoll der X. Arbeitstagung des Arbeitskreises für südwestdeutsche Stadtgeschichtsforschung Calw 12.-14. November 1971, hg. von Erich Maschke/Jürgen Sydow (VKgL B 82), Stuttgart 1974. S. 20-28; Dietmar Willoweit, Die Entwicklung und Verwaltung der spätmittelalterlichen Landesherrschaft, in: Deutsche Verwaltungsgeschichte, Bd.1: Vom Spätmittelalter bis zum Ende des Reiches, hg. von Kurt 
ten Besiedlung der Grafschaft wurden jedoch einige, zumeist kleinere Städte in Ämter eingegliedert, ohne Amtsstadt zu sein, was sich nachteilig auf ihre urbane Entwicklung auswirken musste. Dies lässt sich auch an der Mehrzahl unserer Fälle erkennen. Nachdem die Grafen von Württemberg 1342 die Stadt Tübingen gekauft hatten, wurde Gönningen dem neu gebildeten Tübinger Amt unterstellt. Neustadt fiel an das Amt Waiblingen, Gutenberg an das Amt Kirchheim, Heiningen an das Amt Göppingen und Hohenhaslach sowie Horrheim an das Amt Vaihingen. Neben dem Verlust jeglicher administrativer Funktionen zogen diese Vorgänge auch rechtliche und wirtschaftliche Konsequenzen nach sich. Da nämlich die Amtsstadt zugleich als Amtsgericht fungierte und als solches die hohe Gerichtsbarkeit ausübte, bedeutete dies für untergeordnete Städte den Verlust derselben, was sich sowohl bei Gönningen als auch bei Heiningen beobachten lässt und auch auf alle anderen Beispielstädte, die zuvor die hohe Gerichtsbarkeit besessen hatten, zugetroffen haben dürfte ${ }^{85}$. In Gutenberg etwa wurde nun auch der Schultheiß vom Kirchheimer Vogt eingesetzt, wobei das Gutenberger Gericht nur noch eine Beratungsfunktion inne hatte ${ }^{86}$.

In wirtschaftlicher Hinsicht konnten unsere Städte, die über nur geringes Potential verfügten, gegen die Konkurrenz der bestimmenden und nicht selten allzu nahen Amtsstädte nichts ausrichten, was ihre ohnehin defizitäre Marktsituation weiter beeinträchtigte. Gönningen z. B. verfügte nur noch über einen bedeutungslosen Vieh- und Krämermarkt und hatte den ökonomisch bedeutsameren Wochenmarkt bereits ebenso verloren wie Horrheim, dem gleichfalls nur ein Jahrmarkt blieb, während Gutenberg immerhin von seiner Lage am Albaufstieg in Richtung Ulm und Esslingen profitierte und infolgedessen eine Zollstelle erhielt ${ }^{87}$. Zu den Marktverlusten gesellten sich Pflichtabgaben und andere Benachteiligungen, die eine zusätzliche Belastung darstellten. So mussten die Nichtamtsstädte allein für die Instandhaltung ihrer Wehranlagen aufkommen - im Gegensatz zur Amtsstadt, die dafür die Amtsdörfer heranziehen konnte - und hatten überdies natürlich die jährliche Steuer zu zahlen. Mit 30 Pfund Heller lag Neustadt dabei im Durchschnitt der Nichtamtsstädte - Neubulach etwa führte 60 Pfund Heller ab, während es bei Mün-

G. A. Jeserich/Hans Pohl/Georg-Christoph von Unruh, Stuttgart 1983, S. 66-143, hier S. 81-104; und jüngst Peter Rückert, Von der Stadt zum Amt: Zur Genese württembergischer Herrschafts- und Verwaltungsstrukturen, in: ZWLG 72 (2013) S. 53-73. Speziell zu den Amtsstädten s. Trugenberger (wie Anm.18).

${ }^{85} \mathrm{Zu}$ Gönningen s. Kinkelin (wie Anm.64) S.28. Zu Heiningen s. Ziegler (wie Anm. 23) S. 82.

${ }^{86}$ Heimatbuch Nürtingen (wie Anm. 58) Bd.1, S. 317; Schneider (wie Anm. 29) S. 149; Göтz (wie Anm. 28) S. 35 f.

$87 \mathrm{Zu}$ Gönningen s. OAB Tübingen, S. 382; Kinkelin (wie Anm. 64) S. 28. Zu Horrheim s. Wissmann (wie Anm. 37) S. 109. Zu Gutenberg s. Kurt Steudle, 700 Jahre Gutenberg. Spielball des Schicksals. Ein Streifzug durch die Geschichte, in: Beiträge zur Heimatkunde des Bezirks Kirchheim unter Teck N.F. 42 (1986) S. 61-65, hier S. 62; Göтz (wie Anm. 28) S. 35; Heimatbuch Nürtingen (wie Anm. 58) Bd. 2, S. 301. 
singen nur 24 Pfund Heller und bei Oberriexingen nur 10 Pfund 15 Schilling Heller waren $^{88}$. Bei Horrheim waren es allerdings ganze 120 Pfund Heller und $120 \mathrm{Ohm}$ Wein nach Vaihinger Maß, wie ein Lagerbuch von 1523 verzeichnet ${ }^{89}$. Damit zahlte Horrheim mehr als etwa die Amtsstadt Nürtingen mit 56 Pfund Heller und stieß in die Regionen von Amtsstädten wie Cannstatt mit 110 Pfund Heller vor ${ }^{90}$.Zieht man den Steuerbetrag als Indikator für die wirtschaftliche Entwicklung heran, dürfte Horrheim also trotz aller bereits geäußerten Einschränkungen nicht allzu unvermögend gewesen sein. Tatsächlich war es auch die bevölkerungsreichste Stadt unter unseren Beispielen und verfügte bei der Schatzung von 1470 über 153 Schatzungspflichtige und damit über geschätzte 450 bis 600 Einwohner, während es 1534 bereits 203 Schatzungspflichtige und folglich ca. 600 bis 800 Einwohner waren ${ }^{91}$.

Neben den geschilderten Auswirkungen hatte die Eingliederung der Beispielstädte in die württembergische Amtsstruktur in Verbund mit den Kaufmodalitäten auch zur Folge, dass frühere Herrschaftsverbände zum Teil auseinandergerissen wurden. Die einstige Siedlungsgemeinschaft Heiningen, Gammelshausen, Eschenbach, Lotenberg und Iltishausen z. B. zerfiel, als Agnes von Helfenstein, die Witwe Simons von Teck, ihren Erbteil mit Eschenbach, Iltishausen und einem Teil Lotenbergs an ihre Familie verkaufte und es den Württembergern trotz eines Verzichts Herzog Friedrichs von Teck im Jahr 1352 nicht gelang, die Orte in ihre Verfügungsgewalt zu bringen ${ }^{92}$. Für Heiningen, das ursprünglich von den Herzögen von Teck zum Mittelpunkt dieses Gebiets gemacht worden war, bedeutete dies folglich eine enorme Bedeutungsminderung. Die Zugehörigkeit Gönningens wiederum hatte stets der alten Grafschaft Achalm gegolten und die Stadt orientierte sich daher in östliche Richtung nach Pfullingen. Mit der Zuteilung zum Amt Tübingen geriet Gönningen aber plötzlich in ganz andere räumliche Zusammenhänge und wurde aus seinen früheren Bindungen herausgelöst. Wie eng diese gewesen waren, zeigte sich noch 1634, als Claudia de' Medici, Witwe Erzherzog Leopolds V. von Österreich, Gönningen und seine Nachbarorte als Pfandschaft Achalm für sich beanspruchte ${ }^{93}$.

${ }^{88} \mathrm{Zu}$ Neustadt s. Dietz (wie Anm. 49) S. 87. Die anderen Angaben nach WüSt, S. 192 (Oberriexingen) und 394 (Neubulach); Beschreibung des Oberamts Münsingen, hg. vom K. Statistischen Landesamt, Stuttgart ${ }^{21912, ~ S . ~} 542$ (Münsingen).

89 Zitiert nach Wissmann (wie Anm. 37) S. 107. Einige Seiten später gibt Friedrich Wissmann die widersprüchliche Information, Horrheim habe eine Steuer von 70 Pfund Heller abführen müssen, ohne jedoch einen Beleg zu nennen (ebd., S. 116). Ich will mich daher am Lagerbucheintrag orientieren. Auch 70 Pfund Heller entsprächen aber noch den Summen größerer Nichtamtsstädte bzw. kleinerer Amtsstädte.

$90 \mathrm{Zu}$ den Steuerbeträgen s. WüSt, S. 190 (Nürtingen) und 254 (Cannstatt).

${ }^{91}$ Die Zahlen nach Wissmann (wie Anm. 37) S. 116 und 119. Bei der Annahme von Erich Buhl, Hohenhaslach habe 1559 aufgrund von 43 Taufen über ca. 1.500 Einwohner verfügt, muss es sich um einen Druckfehler und eine Null zu viel handeln (BuHl [wie Anm. 64] S. 57).

92 Ebd., S. 18.

93 Vgl. Kinkelin (wie Anm. 64) S. 12, der darauf hinweist, dass die Bindungen nach Osten zu Zeiten des erfolgreichen Gönninger Samenhandels wiederbelebt wurden. 
Aber nicht nur die Amtszuteilungen sorgten für eine Durchbrechung des früheren gewohnten Umfelds. Auffallend häufig wurden unsere Städte nämlich auch verpfändet und genossen dadurch kaum herrschaftliche Kontinuität. Gönningen etwa gelangte bald nach seinem Verkauf an Württemberg pfandweise an die Herren von Gundelfingen, dann in Teilen an die Grafen von Hohenberg, danach an den Truchsessen Ulrich von Urach und schließlich noch an die Reichsstadt Reutlingen $^{94}$. Im Jahr 1372 wurde es für 1.350 Pfund Heller von Reutlingen ausgelöst, geriet im darauffolgenden Städtekrieg jedoch abermals zu einem Streitpunkt zwischen der Reichsstadt und der Grafschaft. Hohenhaslach und Horrheim fielen zunächst an Schwigger von Gundelfingen und dessen Sohn und kamen 1441 an Wolf von Züllnhart, dessen Verwandter Syfried bereits 1392 Heiningen als Pfand besessen hatte ${ }^{95}$. Hoheneck wurde nach seinem Kauf direkt wieder an die Familie der Hacken von Hoheneck verpfändet und 1432 auf Albrecht Speth übertragen. Herzog Eberhard II. löste es im Jahr 1496 mit der Absicht aus, es an Wolfgang Gotzmann, den Hofmeister seiner Gemahlin, weiterzureichen, was allerdings nicht zustande $\mathrm{kam}^{96}$. Aus der Perspektive der Herrschaft Württemberg war mit den vielmaligen Besitzwechseln in erster Linie die kurzfristige Beschaffung von Geldmitteln bzw. die Befriedigung ihrer Gläubiger verbunden, da mit jeder Pfandschaft in der Regel vorherige Darlehen einhergingen. Da die betrachteten Städte innerhalb des territorialen Aufbaus, wie gesehen, gerade nicht von Bedeutung waren, konnten sie folglich problemlos durch viele Hände gereicht und umso besser in finanzieller Hinsicht nutzbar gemacht werden.

Es wurde bislang deutlich, dass die Beispielstädte innerhalb des württembergischen Territoriums eine sehr untergeordnete Rolle spielten. Aufgrund der äußeren Rahmenbedingungen vermochten sie keinerlei administrative, wirtschaftliche oder rechtliche Funktionen zu erlangen und wurden von ihren neuen Herren bestenfalls als Tauschmittel verwendet. Einen anderen Weg schlugen allerdings die Städte Hoheneck und Ochsenburg ein. Auch Hoheneck diente zwar zunächst als Pfand, wurde aber am Ende des 15. Jahrhunderts, nachdem es württembergisches Eigengut geworden war, zusammen mit dem Nachbarort Neckarweihingen zu einem „Miniaturamt ${ }^{\text {“97 }}$ zusammengefasst. Die hohe Gerichtsbarkeit lag zwar auch hier beim Marbacher Vogt, dem der Hohenecker Schultheiß unterstellt war, jedoch verfügte Hoheneck als Amtsstadt über die Landstandschaft und war infolgedessen nicht nur beim Marbacher Städtetag von 1514 vertreten, sondern auch von 1594-

94 OAB Tübingen, S. 384.

${ }_{95} \mathrm{Zu}$ Hohenhaslach und Horrheim s. Wissmann (wie Anm. 37) S.96f. Zu Heiningen s. Bauer (wie Anm. 40) S. 18.

96 Felden (wie Anm. 27) S. 102 und $109 \mathrm{f}$.

97 LBW, Bd. 3, S. 426. Vgl. auch die Karte zu den württembergischen Ämtern um 1525 in: Landschaft, Land und Leute. Politische Partizipation in Württemberg 1457 bis 2007. Begleitbuch und Katalog zur Ausstellung des Landesarchivs Baden-Württemberg, Hauptstaatsarchiv Stuttgart und des Landtags von Baden-Württemberg, bearb. von Peter RückerT, Stuttgart 2007, S. 115. 
1608 regelmäßiges Landtagsmitglied ${ }^{98}$. Als das Herzogtum Württemberg im Jahr 1519 vom Schwäbischen Bund eingenommen wurde, musste es aufgrund des geleisteten Widerstands ein Schutzgeld von 50 Gulden zahlen und verlor in der Folge seine Sonderstellung ${ }^{99}$. Der zurückgekehrte Herzog Ulrich von Württemberg annullierte diese Entscheidung im Jahr 1534 jedoch und bestätigte das Amt Hoheneck. Die folgenden Jahrzehnte waren durch Konflikte geprägt, einerseits mit Marbach, das immer wieder versuchte, denStatus des ungeliebten, kleinen Nachbaramtes zu untergraben, andererseits mit dem Amtsort Neckarweihingen, der weder zum wiedererrichteten Schießhaus noch zur Hohenecker Stadtmauer finanziell beitragen wollte. Die Neckarweihinger beschwerten sich, dass Hoheneck so unzureichend befestigt sei, dass man in Kriegszeiten ohnehin nicht dort würde Schutz suchen wollen, und dass eine solche Beihilfe zuvor auch nicht gefordert worden $\operatorname{sei}^{100}$. Offensichtlich hatte Hoheneck also trotz seiner Stellung strukturell keineswegs an andere Amtsstädte anknüpfen können und wurde von seinem Amtsort gar als Fleck verunglimpft - was zwar gewiss lediglich die Neckarweihinger Argumentation stützen sollte, jedoch auch eine nur eingeschränkte städtische Entwicklung andeutet. Nichstdestoweniger gewann Hoheneck aber aufgrund seines Amtes zentrale Funktionen, unter anderem mit der 1609 errichteten Kellerei ${ }^{101}$, eine geringfügige politische Mitsprache durch die Landstandschaft sowie insgesamt einen festen Platz im württembergischen Herrschaftsgefüge. Diesen Aufschwung belegen auch die zunehmenden Einwohnerzahlen: Hatten im Hoheneck des 14. Jahrhunderts noch ca. 200-300 Menschen gelebt, so waren es im 16. Jahrhundert bereits $400-500^{102}$. Hoheneck war damit nach Horrheim die in demographischer Hinsicht größte unserer Beispielstädte. Im Vergleich verfügten Neustadt und Gutenberg im frühen 16. Jahrhundert nur über ca. 250 bzw. ca. 170 Einwohner ${ }^{103}$. Das langsame Ende des Amtes Hoheneck trat erst an der Wende vom 17. zum 18. Jahrhundert ein. Bereits 1672 stand es kurz davor, aufgelöst zu werden, weil sich infolge des DreiBigjährigen Kriegs eine horrende Schuldenlast angesammelt hatte. Als im Jahr 1716 aber der letzte Hohenecker Keller seinen Sitz ins neu errichtete Ludwigsburg ver-

${ }^{98}$ Zur Gerichtsbarkeit s. Stein (wie Anm. 27) S. 136. Zum Marbacher Städtetag s. Württembergische Landtagsakten 1498-1515, hg. von Wilhelm OHR/Erich KовеR (Württembergische Landtagsakten, Bd.1/1), Stuttgart 1913, Nr.44, S.139-145. Zur Landstandschaft s. Schaав (wie Anm. 9) S. 225 mit Anm. 24; Stein (wie Anm. 27) S. 136.

99 Felden (wie Anm. 27) S. 112 und 202; Walter Grube, Hoheneck und Neckarweihingen im Kriegsjahr 1519, in: Schwäbische Heimat 19 (1968) S.72-75, hier S.72; Stein (wie Anm. 27) S. 132.

${ }_{100} \mathrm{Zu}$ den Konflikten mit Marbach und Neckarweihingen vgl. Felden (wie Anm. 27) S. 202; Stein (wie Anm. 27) S. 42f. und 133-135.

101 Vgl. Felden (wie Anm. 27) S. 101.

102 Die Zahlen nach Stein (wie Anm. 27) S. 166.

103 Die Zahlen nach Dietz (wie Anm. 49) S. 9 und 22; Götz (wie Anm. 28) S. 48. 
legte, war es schließlich so weit: Nur drei Jahre später wurde das Amt Hoheneck aufgelöst und in das Oberamt Ludwigsburg eingegliedert ${ }^{104}$.

Eine gänzlich andere Entwicklung nahm das im Zabergäu gelegene Ochsenburg, das ursprünglich einmal Ochsenberg geheißen hatte, sich aber 1593 in Ochsenburg umbenannte, 1815/16 wieder zu Ochsenberg zurückkehrte und sich 1899 dann endgültig für Ochsenburg entschied ${ }^{105}$. Nachdem es 1360 wahrscheinlich durch das Vermächtnis des Grafen Heinrich von Vaihingen an Württemberg gelangt war, wurde es in der Folge einige Male als Lehen ausgegeben, unter anderem an die Herren von Enzberg und die Herren von Urbach ${ }^{106}$. Im Jahr 1385 erhielt schließlich Hennel I. von Sternenfels, der den Grafen als württembergischer Vogt im Zabergäu diente, erst einen Teil und 1392 ganz Ochsenburg als Lehen. Seine Familie war hier bereits um 1340 begütert und tatsächlich sollte Ochsenburg bis 1749 als Lehen in ihrem Besitz bleiben ${ }^{107}$. Während dieser Zeit wird Ochsenburg wiederholt als Stadt erwähnt, so nämlich in den Jahren 1481, 1573, 1593 und $1736^{108}$. Gleichwohl dürfte diese Stadt, die im 17. Jahrhundert nur über 40 bis 50 Einwohner verfügt haben soll ${ }^{109}$, von völlig anderem Zuschnitt gewesen sein. Ochsenburg wurde nämlich unter den Herren von Sternenfels über den Ritterkanton Kraichgau besteuert und war damit fast völlig aus dem württembergischen Herrschaftsverband ausgegliedert.

Wie Gerhard Fouquet zeigt, waren solche ritterschaftlichen Kleinstädte innerhalb ihrer eng umgrenzten Gebiete zwar stets mit zentralen Aufgaben betraut, verfügten jedoch über eine nur eingeschränkte soziale und wirtschaftliche Entwicklung bei gleichzeitiger großer Abhängigkeit von den niederadligen Stadtherrn, nur wenigen persönlichen Freiheiten und einer stark agrarischen Prägung ${ }^{110}$. Entsprechend machten auch die Herren von Sternenfels Ochsenburg zu ihrem Herrschaftsmittelpunkt: Sie residierten im mehrfach renovierten und erweiterten Ochsenburger Schloss und errichteten 1564 ein Kellereigebäude sowie einen Fruchtkasten ${ }^{111}$. Die Abgeschlossenheit Ochsenburgs scheint den württembergischen Lehnsherren jedoch bisweilen missfallen zu haben: Im zweiten Drittel des 16. Jahrhunderts kam es zu einem Rechtsstreit bezüglich der hohen Gerichtsbarkeit, die von den Herren von Sternenfels ausgeübt wurde, und man einigte sich schließlich darauf, dass

${ }^{104} \mathrm{Zu}$ den Ereignissen s. Stein (wie Anm. 27) S. 137 f.; Felden (wie Anm. 27) S. $202 \mathrm{f}$.

105 Assfahl (wie Anm. 82) S. 54.

106 Vgl. ebd., S. 53; Klunzinger (wie Anm. 82) S.133, 135 und 230; Beschreibung des Oberamts Brackenheim, hg. von dem königlichen statistisch-topographischen Bureau, Stuttgart/Tübingen 1873, ND Magstadt 1976 (künftig: OAB Brackenheim), S. 385.

${ }_{107} \mathrm{Zu}$ den Herren von Sternenfels zwischen 1385-1749 s. Assfahl (wie Anm. 82) S. 77f.; Klunzinger (wie Anm. 82) S. 213-222; OAB Brackenheim, S. 385.

$108 \mathrm{Zu} 1573$ s. Assfahl (wie Anm. 82) S. 54. Zu den anderen Daten s. OAB Brackenheim, S. 384 .

109 KB Heilbronn, Bd. 2, S. 518.

110 Fouquet (wie Anm. 60) bes. S. 73, 104-109 und 120.

111 Assfahl (wie Anm. 82) S. 78. 
Württemberg ein Mitspracherecht erhalten solle ${ }^{112}$. Mehr erfahren wir über die inneren Ochsenburger Verhältnisse leider nicht. Erst im Jahr 1749 kam Ochsenburg wieder an Württemberg zurück, als die Herren von Sternenfels ihr Lehen für 308.500 Gulden verkauften. Nachdem es für weitere 115.000 Gulden aus dem Besteuerungsrecht des Ritterkantons Kraichgau ausgelöst worden war, erhob Württemberg es zusammen mit den Orten Leonbronn, Zaberfeld und Michelbach zu einem Kammerschreibereigut ${ }^{113}$.

Wie zu sehen war, stellt der Übergang an die Grafschaft Württemberg eine bedeutsame Zäsur in der Geschichte unserer Fallbeispiele dar. Die nur mäßig entwickelten Städte, die innerhalb ihrer früheren Herrschaften noch von relativer Bedeutung gewesen waren, versanken, einmal in das große württembergische Territorium eingegliedert, in administrativer, rechtlicher und wirtschaftlicher Bedeutungslosigkeit - ein Prozess, den nur Hoheneck und Ochsenburg aufgrund ihrer Sonderentwicklungen vermeiden konnten. Wie sich die skizzierten Vorgänge auf den Stadtstatus auswirkten, wird nun Thema des letzten Untersuchungsabschnitts sein.

\section{Von der Stadt zum Dorf: Der Verlust des Stadtstatus}

Wann eine Stadt ihre städtische Qualität verlor, lässt sich zeitlich kaum terminieren, weil mit diesem Vorgang kein offizieller, gar schriftlich festgehaltener Rechtsakt verbunden war, der einen einzigen Moment hätte herausdeuten können. Stattdessen gleicht der Statusverlust in dieser Hinsicht dem Prozess der Stadtwerdung, der sich verbindlichen Angaben ebenso entzieht und nur durch eine intensive Spurensuche nachvollziehbar wird. Datierungsversuche wurden dennoch unternommen und fielen häufig, je nach Gutdünken des Verfassers, recht großzügig aus. Im Falle Gutenbergs etwa führte man den Verlust des Stadtstatus auf den Dreißigjährigen Krieg zurück, als die Stadt nur noch über 27 Einwohner verfügte, oder sogar erst auf die Mitte des 18. Jahrhunderts ${ }^{114}$. Betrachtet man jedoch die wenigen vorhandenen Indizien, so scheint Gutenberg bereits wesentlich früher zu einem Dorf herabgesunken zu sein. Als im Jahr 1918 Ausgrabungen im Bereich der ehemaligen Stadtmauer vorgenommen wurden, veranlassten die Befunde den Grabungsleiter Friedrich Hertlein zu der Annahme, dass die Mauern wahrscheinlich schon um die Mitte des 16. Jahrhunderts ihre Bedeutung verloren hatten. Die Südmauer der Gutenberger Pfarrkirche schien nämlich ein umgebautes Stück der Stadtbefestigung gewesen zu sein, der Kirchturm gar ein alter Befestigungsturm.

112 OAB Brackenheim, S. 386.

113 Ebd.

114 Vgl. Friedrich Hertuein, Die ehemalige Stadt Gutenberg (OA Kirchheim), in: Blätter des Schwäbischen Albvereins 31 (1919) Sp.7-11, hier Sp. 9; STeudle (wie Anm. 87) S.63; Mayer (wie Anm. 43) S. 12. 
Und tatsächlich ist auch im „Seebuch“ des Jakob Ramminger von 1596 bei der Darstellung Gutenbergs keine Stadtmauer mehr zu erkennen ${ }^{115}$. Natürlich weiß man heute, dass die städtische Ummauerung allein nicht das konstitutive Merkmal einer Stadt ist, dessen Fehlen zwingend einen nichtstädtischen Siedlungscharakter anzeigt. Doch andere Anzeichen, die bereits anfänglich erwähnt wurden, stützen Hertleins These. Im Laufe des 16. Jahrhunderts nämlich vollzog sich ein Benennungswandel und Gutenberg wurde im Jahr 1510 nur noch als Dorf bezeichnet ${ }^{116}$.

Die trügerische Aussagekraft einzelner Siedlungsbezeichnungen haben wir freilich schon kennengelernt; allerdings enthält ein Lagerbuch von 1561 den Eintrag, dass Gutenberg vor Jahren ain Stetlin gewesen ${ }^{117}$ sei und auch noch derzeit Stadtrecht habe, worauf sich die mangelnden leibherrlichen Abgaben der Einwohner zurückführen ließen. Der Chronist Daniel Wolleber schrieb knappe 30 Jahre später, dass Gutenberg aufgrund seiner Privilegia Und alte gemeur einst ein Stättlin gewesen sei $^{118}$. Und das bereits erwähnte „Seebuch“ des Jakob Ramminger ließ nicht nur die Gutenberger Stadtmauer vermissen, sondern auch das städtische Wappen, das bei keiner anderen Stadt fehlte ${ }^{119}$. Gutenberg muss seinen Stadtstatus also bereits im laufenden 16. Jahrhundert verloren haben und nicht erst im 17. oder 18. Jahrhundert ${ }^{120}$.

Sehr interessante Vorgänge werden im Falle Heiningens erkennbar. Die ehemals teckische Stadt startete nämlich im späten 15. Jahrhundert den Versuch, sich ihr altes königliches Marktprivileg erneuern zu lassen. Graf Eberhard V. von Württemberg kam dieser Bitte nach und bestätigte die ihm vorgelegte Königsurkunde von 1284 ohne viel Federlesens. Im Jahr 1519 aber wandten sich die Heininger für eine erneute Bestätigung an Freiburg selbst, erkundigten sich dabei zugleich nach den Inhalten der gewährten Freiheiten und erhielten als Antwort einen Auszug mit 37 Punkten ${ }^{121}$. Diese Geschehnisse erwecken den Eindruck, als hätten die Heininger ihrem alten Stadtrecht neues Leben einhauchen und sich zur Sicherheit bei den Freiburgern selbst bezüglich der konkreten Inhalte vergewissern wollen. Die Zusammenhänge erscheinen jedoch in einem anderen Licht, wenn man sich auch bei Heiningen den noch deutlicher als bei Gutenberg hervortretenden Benennungswandel vor Augen führt. Schon 1352 nämlich wird Heiningen als Dorf und in Zusammenhang mit der Anfrage bei Graf Eberhard V. 1489 als Markt erwähnt, 1590 bei Daniel Wolleber wieder als Dorf und 1594 wieder als Markt ${ }^{122}$. Wenn wir diese Angaben mit der Tatsache kombinieren, dass der Wochenmarkt Heiningens

$115 \mathrm{Zu}$ den Ausgrabungen von 1918 s. Hertlein (wie Anm. 114) Sp.7f. Vgl. auch Götz (wie Anm. 28) S. 22 f. und 34.

116 Ebd., S. 22.

117 Zitiert nach ebd., S. 22.

118 Zitiert nach ebd., S. 25.

119 Ebd., S.22f. und 34.

120 S. zur früheren Datierung auch LBW, Bd. 3, S. 200.

121 Ziegler (wie Anm. 23) S. 77. Vgl. auch Götz (wie Anm. 63) S. 130.

122 Ziegler (wie Anm. 23) S. 80 und 84. 
lange Zeit Bestand hatte und noch 1714 wieder bestätigt wurde, wird es den Heiningern bei diesen Erneuerungen nicht um eine Wiederbelebung ihres Stadtstatus gegangen sein, der schon nach 1321, mit dem Übergang an Württemberg, nicht mehr zum Tragen $\mathrm{kam}^{123}$. Stattdessen dürfte ihnen die Bekräftigung desjenigen Privilegs wichtig gewesen sein, das ihre derzeitige Stellung untermauerte, nämlich des Marktprivilegs Rudolfs von Habsburg. Dessen wiederholte Erneuerung im Jahr 1519 wurde notwendig, weil nach der Eroberung Württembergs durch den Schwäbischen Bund die Landesherrschaft wechselte und sich Heiningen auch für künftige Zeiten absichern wollte. Entsprechend wird auch im Göppinger Vogtbericht von 1535 nur davon berichtet, dass Heiningen von König Rudolf ein Marktrecht erlangt habe ${ }^{124}$. Kurzum: Im 15. bzw. 16. Jahrhundert war der einstige Stadtstatus längst vergessen und Heiningen konzentrierte sich nun mehr auf seine Position als Markt ${ }^{125}$.

Dass Horrheim eine relative wirtschaftliche Leistungsfähigkeit entwickelt hatte, wurde bereits angesichts der abzuführenden gewöhnlichen Steuer und der zunehmenden Einwohnerzahl deutlich. Diese Tendenz schien sich auch im frühen 16. Jahrhundert fortzusetzen. Für das Jahr 1514 erfahren wir, dass der Ort sich in den letzten Jahren mit Befestigungen versehen habe ${ }^{126}$. Dabei dürfte es sich um eine Erweiterung oder eine Erneuerung gehandelt haben, da bereits für das Jahr 1304 eine Befestigung belegt ist, die möglicherweise nur ein Provisorium war. Jedenfalls schien Horrheim imstande zu sein, die damit verbundenen Kosten zu tragen. Fünf Jahre später sah es sich durch die Truppen des Schwäbischen Bundes anlässlich der Eroberung Württembergs gezwungen, innerhalb von 14 Tagen eine Summe von 600 Gulden aufzubringen. Dies überstieg jedoch die Mittel des geplünderten und gebrandschatzten Ortes bei weitem, der daraufhin das Stift Speyer um Hilfe ersuchte ${ }^{127}$. Ob das Strafgeld dabei in einem gewissen Zusammenhang mit der vorhandenen Vermögenslage gesehen werden kann, ist freilich unsicher. War Horrheim im frühen 16. Jahrhundert aber immer noch eine Stadt? Wie bei Gutenberg und Heiningen sprechen auch hier die Quellen eine eindeutige Sprache. Schon 1439 wird Horrheim nur noch als Dorf bezeichnet; als Stadt begegnet es im gesamten 15. Jahrhundert nicht mehr ebenso wenig wie in den Lagerbüchern des 16. Jahrhunderts, die für das Jahr 1523 etwa nur mehr den Dorfgraben anstelle des Stadtgrabens aufführen ${ }^{128}$. Neben diesem Benennungswandel deutet sich zudem ein Strukturwandel an: Im Jahr 1493 nämlich entschied Graf Eberhard V., dass den Orten Hohenhaslach, Horrheim und Ensingen wegen Wildschadens der Schweinhaber

123 Ebd., S. 84; Bauer (wie Anm. 40) S. 65; LBW, Bd. 3, S. 334.

124 Ziegler (wie Anm. 23) S. 77; Götz (wie Anm. 63) S. 130, Anm. 157.

125 Als weiteres Beispiel für einen Statusverlust trotz königlicher Privilegierung nennt Adolf Layer die ehemalige Stadt Buchloe in Ostschwaben: Layer (wie Anm. 4) S. 9 f.

126 Orth/Kies (wie Anm. 31) S. 40, Anm. 101.

127 Wissmann (wie Anm. 37) S. 126.

128 WR 14284; Wissmann (wie Anm. 37) S. 17. 
nachgelassen werden solle und die Bauern künftig ausgediente Jagdhunde halten dürften, um das Wild aus ihren Gütern zu verjagen. Wenngleich es sich hier nur um eine einzelne Nachricht handelt, so dürfte sie doch als Hinweis zu sehen sein, dass Hohenhaslach und Horrheim mittlerweile stark agrarisch geprägt waren und sich mehr und mehr Bauern ansiedelten - eine Veränderung, die auch Roland Flückiger-Seiler bei den „Statuswüstungen“ der Basse-Gruyère beobachten konnte ${ }^{129}$. Insgesamt schätzt man, dass Hohenhaslach und Horrheim ihren Stadtstatus schon bald nach ihrem Übergang an Württemberg im Jahr 1360 verloren hatten, ohne dass sich dies in den Quellen deutlicher wiederspiegeln würde ${ }^{130}$.

Die Gründung Neustadts bei Waiblingen offenbarte eine Vielzahl kursierender Thesen bezüglich des Stadtgründers und seiner Erwägungen. Nachdem das Städtchen per königlichem Beschluss 1298 an Württemberg gelangt war, nahm es allerdings eine ganz unauffällige Entwicklung. In den Jahren 1304 und 1350 wird es als nova civitas bzw. als Stadt erwähnt, erhielt um 1320 die Martinskirche, die eine Filialkirche von Waiblingen darstellte, und musste zur Jahrhundertmitte eine Pestwelle erleben ${ }^{131}$. Als Überbleibsel seiner einstigen Stellung als Stadt erfährt man aus einem Lagerbuch von 1568/69, dass seine Einwohner, wie in anderen Städten, nicht dem Hauptrecht unterlagen ${ }^{132}$. Wann es allerdings zum Dorf herabsank, lässt sich mit dem laufenden 14. Jahrhundert nur ebenso grob schätzen wie bei Gönningen, das ähnlich still und leise seine städtische Geschichte beendete ${ }^{133}$. Im Jahr 1329 letztmalig als Stadt erwähnt, dürfte der Verlust seines städtischen Ranges wohl wie bei Neustadt in Verbindung mit dem Übergang an Württemberg zu sehen sein ${ }^{134}$.

129 Flückiger-Seiler bezeichnet die Umstrukturierung der Bevölkerung als ersten Schritt eines Umwandlungsprozesses von der Stadt zum Dorf, auf den eine bauliche Veränderung der Siedlung folge: Flǘckiger-Seiler (wie Anm. 8) S. 178 und 187. Vgl. auch Ernst (wie Anm.7) S. 127: „Der eigentliche Nährboden einer Stadt war die Umgebung, soweit sie zur gleichen Herrschaft gehörte. Das reichte oft nicht aus, um eine Stadt in Blüte zu bringen; die neue Stadt, die mit großen Hoffnungen angefangen war (!), ist dann rasch verkümmert; in die engen Gassen, die für ganz andere Zwecke angelegt waren, zogen Bauern und machten sie für ihre Zwecke zurecht." Zu Hohenhaslach und Horrheim s. BuHL (wie Anm. 64) S. 51 f.

130 Vgl. zu Hohenhaslach LBW, Bd. 3, S. 449; OAB Vaihingen, S. 165. Vgl. zu Horrheim LBW, Bd. 3, S. 463; Wissmann (wie Anm. 37) S. 14. Vgl. auch Scheuerbrandt (wie Anm. 9) S. 158.

131 LBW, Bd.3, S. 561; Dietz (wie Anm. 49) S. 2; Haupt (wie Anm. 47) S. 132; Peterke (wie Anm. 54) S. 45.

132 Dass diesbezüglich 1298 eine spezielle Absprache getroffen worden sein könnte, wie Joachim Peterke meint (Peterke [wie Anm. 54] S. 49f.), halte ich für zweifelhaft.

${ }^{133}$ Zur Datierung des Neustadter Statusverlusts s. Weller (wie Anm.7) S. 336; DietZ (wie Anm. 49) S. 5; Peterke (wie Anm. 54) S. 45. Zur Datierung des Gönninger Statusverlusts s. Handbuch der historischen Stätten Deutschlands, Bd.6: Baden-Württemberg, hg. von Max Miller/Gerhard Taddey (Kröners Taschenausgabe, Bd.276), Stuttgart ${ }^{2} 1980$, S. 260.

134 Vgl. Ackermann (wie Anm.26) S.11; Kinkelin (wie Anm.64) S.11; LBW, Bd.7, S. 64 . 
Hoheneck hatte es als einzige der hier betrachteten Städte zur württembergischen Amtsstadt gebracht und diese Stellung mehr als zwei Jahrhunderte lang bewahren können. Jedoch stand es während des Dreißigjährigen Krieges kurz vor seiner totalen Zerstörung. Hatten bereits zahlreiche Einquartierungen von Kompanien in den 1620er-Jahren hohe, kaum mehr zu tragende Kosten verursacht, ereignete sich schließlich im Jahr 1634 die Katastrophe. Nach der Schlacht von Nördlingen wurde Hoheneck so vollkommen geplündert und niedergebrannt, dass Schäden in Höhe von knapp 120.000 Gulden entstanden. Nachdem infolge eines solchen Vernichtungswerks und seiner Begleiterscheinungen viele aus dem Ort geflohen waren, zählte Hoheneck im Jahr 1638 nur noch ca. 20 Einwohner. Der langsame Wiederaufbau, der in den folgenden Jahrzehnten erfolgte und von hohen Schulden begleitet war, kam durch Franzoseneinfälle am Ausgang des 17. Jahrhunderts erneut ins Stocken, so dass sich auch 1698 nicht mehr als 13 Einwohner in Hoheneck aufhielten. Erst knappe 20 Jahre später hatte sich die Einwohnerzahl erholt und war auf knapp 300 gestiegen ${ }^{135}$. Amtsmittelpunkt blieb Hoheneck allerdings bis zu seiner Eingliederung ins Oberamt Ludwigsburg im Jahr 1719 und es ist sehr wahrscheinlich, dass seine städtische Qualität, von der freilich nach den Kriegszerstörungen des 17. Jahrhunderts nicht mehr allzu viel übrig geblieben sein dürfte, zumindest nominell mit diesem Status verbunden war und folglich erst im 18. Jahrhundert verloren ging. Auf diesen Zusammenhang weist auch Meinrad Schaab hin, nach dem Hoheneck erst nach dem Verlust des Amtssitzes nicht mehr als Städtlein galt und daher im Staatshandbuch von 1775 auch nicht mehr an der entsprechenden Stelle auftaucht ${ }^{136}$.

Einen ähnlichen Niedergang erlebte Ochsenburg, das wie Hoheneck einen Sonderweg eingeschlagen hatte. Nachdem die Herren von Sternenfels es 1749 an Württemberg zurückgegeben hatten, wurde es nach der Ablösung aus dem Ritterkanton Kraichgau zusammen mit Leonbronn, Zaberfeld und Michelbach zu einem Kammerschreibereigut mit Stabsamtmann, Keller und Landstandschaft erhoben. Jedoch währte diese Stellung nicht lange. In den Jahren 1806/07 wurde das Stabsamt wieder aufgelöst und Ochsenburg kam zuerst an das Oberamt Güglingen und später an das Oberamt Brackenheim. Doch damit nicht genug: Ab 1814 verkaufte Württemberg alle staatlichen Güter, so dass in der Folgezeit sogar das Schloss, der jahrhundertelange Sitz der Herren von Sternenfels, abgebrochen wurde ${ }^{137}$. Wenig überraschend erscheint Ochsenburg daraufhin im Staatshandbuch von 1824 nur noch als Dorf ${ }^{138}$.

Wenn wir nun als letztem Schritt der Analyse nach den Gründen für die Statusverluste fragen, wird angesichts der zahlreichen Entwicklungsschritte und ihrer

$135 \mathrm{Zu}$ den Ereignissen des 17. Jahrhunderts vgl. Felden (wie Anm. 27) S. 116-122; Stein (wie Anm.27) S. 46 f.

136 Schaab (wie Anm. 9) S. 225. Vgl. auch Felden (wie Anm. 27) S. 99.

137 Vgl. insgesamt Assfahl (wie Anm. 82) S. 53 f.; OAB Brackenheim, S. 386.

138 Assfahl (wie Anm. 82) S. 54. 
Begleitumstände schnell klar, dass es sich um ein ganzes Bündel von Ursachen handelt. So verfügten alle Beispielstädte über schlechte strukturelle Voraussetzungen, was die Verkehrslage und die geographischen Gegebenheiten anbelangte. Steile Berghänge wie bei Gönningen und Gutenberg, Spornlagen wie bei Neustadt und Hohenhaslach und ein zu geringes Hinterland wie bei Horrheim stellten von vornherein Hemmnisse für die städtische Entwicklung dar und mussten sich vor allem auf die wirtschaftliche Situation negativ auswirken ${ }^{139}$. Zudem brachte es die hohe Städtedichte mit sich, dass sich nicht selten in unmittelbarer Nähe ältere, etablierte Städte befanden. Mit Göppingen konnte Heiningen nicht konkurrieren, Hoheneck nicht mit Marbach, Horrheim nicht mit Vaihingen und Neustadt nicht mit Waiblingen, so dass auch hier der städtischen Entwicklung bereits Grenzen gesetzt waren $^{140}$. Die vorherrschenden territorialpolitischen Motive konnten diese Nachteile nicht aufwiegen, zumal erstere mit dem Übergang an Württemberg nicht mehr griffen. Als nachteilig erwies es sich auch bei Gutenberg, Heiningen, Hohenhaslach und Horrheim, dass ihre Gründerfamilien finanziell bereits stark beeinträchtigt waren, ihre Städte kaum zu fördern vermochten und mit den Resten ihrer einstmals größeren Herrschaften einen zu kleinen Rahmen für eine städtischen Entfaltung boten ${ }^{141}$. Thomas Küntzel macht zudem darauf aufmerksam, dass gerade die aus Burgsiedlungen hervorgegangenen Städte wie Gönningen, Gutenberg oder Hoheneck zu einer Risikogruppe der „Statuswüstungen“ zählten, weil mit dem Bedeutungsverlust der Burgen nicht selten auch der Bedeutungsverlust der Stadt einherging ${ }^{142}$.

Wie zu sehen war, markierte der Übergang an Württemberg in Verbund mit dem Verlust der ursprünglichen Stadtgründer eine tiefgreifende Zäsur in der Geschichte unserer Beispielfälle. Diese mussten jetzt nicht nur ohne die „väterliche Pflege“ auskommen, wie es Viktor Ernst ausdrückte ${ }^{143}$, sondern sahen sich mit gänzlich neuen Bedingungen konfrontiert. Nach ihrem eher beschaulichen Dasein als kleine Herrschaftsmittelpunkte wurden sie nun Teil der Grafschaft Württemberg, deren zahlreiche Städte in ein Verwaltungssystem eingebunden waren, das mit seiner Un-

139 Als Parallelbeispiele lassen sich die schweizerischen Städte Rheinau, Elgg, Greifensee und Grüningen sowie das ostschwäbische Ottobeuren anführen, die unter ähnlich suboptimalen Bedingungen gegründet worden waren: Ammann, Waadt (wie Anm. 8) S. 77; Layer (wie Anm. 4) S. $14 \mathrm{f}$.

140 Auch hier finden sich mit Gottlieben, Bieberstein und Wallensen, die jeweils ihren Nachbarn Konstanz, Aarau und Duingen unterlagen, Parallelbeispiele: Ammann, Waadt (wie Anm. 8) S. 77; Stephan (wie Anm. 8) S. 108f. Ähnliche Tendenzen ergaben sich auch in anderen sehr dicht besiedelten Gegenden wie der Basse-Gruyère (FLÜCKIGER-SEILER [wie Anm. 8] S. 185).

${ }^{141} \mathrm{Zu}$ Gutenberg s. Heimatbuch Nürtingen (wie Anm.58) Bd.2, S.300; LBW, Bd.2, S. 300. Zu Heiningen s. Bauer (wie Anm. 40) S.16. Zu Hohenhaslach und Horrheim s. Scheck (wie Anm.65) S.50f.; Scheuerbrandt (wie Anm. 9) S. 158; Buhl (wie Anm. 64) S. 43; Wissmann (wie Anm. 37) S. 92.

142 KüntZel (wie Anm. 9) S. 125.

143 Ernst (wie Anm.7) S. 133. 
terscheidung in Amtsstädte und Nichtamtsstädte mehr und mehr zu einer hierarchischen Abstufung unter den Städten führte. Und da sich außer Hoheneck keine unserer Städte als Amtssitz eignete, nahmen sie in dieser Hierarchie einen sehr weit unten angesiedelten Platz ein und durften in Bezug auf städtische Belange kaum auf die Unterstützung ihrer neuen Stadtherren hoffen. Der in diesem Zusammenhang nicht selten geäußerte Vorwurf, die Grafen von Württemberg hätten Städte wie Neustadt oder Hohenhaslach bewusst demontieren wollen bzw. auf eine Dezimierung ihrer Städte hingezielt, wird sich indes nicht halten lassen ${ }^{144}$. Ganz abgesehen davon, dass eine derart strategische Planung eher nicht den mittelalterlichen Verhältnissen entsprach, konnten die Landesherren angesichts des rasch ansteigenden Städtebestands nicht allen Städten eine Förderung angedeihen lassen und favorisierten diesbezüglich die größeren, potenteren und strategisch wichtigeren unter ihnen, was zu Lasten des Rests ging.

Mit der Eingliederung in das Ämtersystem waren für unsere Städte gravierende Verluste städtischer Kompetenzen verbunden, die bei Gönningen, Gutenberg, Horrheim, Hohenhaslach und Heiningen auf direktem Wege zum städtischen Niedergang führten, der denn auch bald nach dem Übergang an Württemberg erfolgt sein dürfte. Gönningen und Heiningen büßten ihre Hochgerichtsbarkeit ein, Gutenberg seine Eigenschaft als herrschaftliche Residenz, Neustadt den eigentlichen Sinn seiner Gründung und Horrheim seinen Wochenmarkt ${ }^{145}$. Die ohnehin in ihrer Entwicklung noch nicht gefestigten Städte konnten den Verlust der geringfügigen Funktionspalette, die sie sich bereits angeeignet hatten, nicht kompensieren. Verstärkt wurden diese Prozesse durch die Herauslösung aus den früheren Herrschaftsverbänden, eine schnelle Abfolge wechselnder Pfandnehmer und die Konkurrenz mächtigerer Nachbarstädte, deren Vorrangstellung als Amtsstadt noch dazu landesherrlich legitimiert war. Einzig Hoheneck und Ochsenburg gelang es, ihren Stadtstatus für längere Zeit zu konservieren. Während Hoheneck zum Amtssitz aufstieg und dadurch seine zentralen Funktionen bewahrte bzw. ausbaute, konnte Ochsenburg als ritterschaftliche Kleinstadt gerade durch seine Distanz zur württembergischen Herrschaftsstruktur als Stadt bestehen. Bezeichnenderweise besiegelte aber auch in diesen beiden Fällen der Verlust der administrativen Zentralität das städtische Schicksal.

Die zerstörerische Wirkung von Kriegserscheinungen schließlich wurde im Fall Hohenecks thematisiert, das während des Dreißigjährigen Krieges massive Schäden hinnehmen musste und schon beinahe seinem völligen Untergang entgegensah. Die Plünderungen und Brandschatzungen schädigten die Siedlungstopographie, führten zu einem Einwohnerschwund und hatten so langfristige wirtschaftliche Belastungen zur Folge, dass der Stadt die (Über-)Lebensgrundlage völlig entzogen

144 Zu Neustadt s. Seigel (wie Anm. 18) S. 179. Zu Hohenhaslach s. Buhl (wie Anm. 64) S. 48.

${ }^{145}$ Zum Verlust zentraler Funktionen s. das Parallelbeispiel Jagow bei Enders (wie Anm. 8) S.113 f. Vgl. auch Engel (wie Anm. 4) S. 14. 
wurde ${ }^{146}$. Einzig die bleibende administrative Funktion, die der Krieg nicht zu beeinflussen vermochte, hielt den Stadtstatus am Leben, der sich allerdings innerhalb der jahrhundertelang ausgeformten württembergischen Verwaltungsstruktur wahrscheinlich als konstitutives Merkmal an den Amtssitz geheftet hatte. War man Amtssitz, dann war man Stadt, ganz unabhängig von Form und Entwicklung des städtischen Wesens ${ }^{147}$. Auch für Gönningen wurden Kriegszerstörungen und ihre wirtschaftlichen Implikationen als Gründe für das Absinken der Stadt erwogen, nachdem der Ort im späten 14. Jahrhundert in die Auseinandersetzungen zwischen Württemberg und den Reichsstädten, besonders der Reichsstadt Reutlingen, geraten war $^{148}$. Im Vergleich wird jedoch deutlich, dass die direkten Einwirkungen kriegerischer Handlungen eine andere, greifbarere Qualität besitzen als die bisher genannten Ursachen für „Statuswüstungen“. Thomas Küntzel hat diesbezüglich äußere Ursachen, die sich durch eine direkte, zeitlich zumeist eingrenzbare Wirkung auszeichnen, und strukturelle Hintergründe unterschieden, die ihre Wirkung gewissermaßen im Verborgenen entfalteten, dabei aber als eigentliche Katalysatoren das Schwinden des Stadtstatus herbeiführten. Während zu den äußeren Ursachen neben Fehden und Naturkatastrophen auch Kriege zu zählen sind, beziehen sich strukturelle Hintergründe auf veränderte topographische, demographische oder politische Entwicklungen ${ }^{149}$. Die württembergischen „Statuswüstungen" waren nach dieser Systematik vorrangig von strukturellen Hintergründen, also Langzeitfaktoren, beeinflusst. Dies erklärt schließlich auch, warum sich die rückläufigen Prozesse nur mit Mühe greifen lassen und warum man meist nur ein Einschlafen des Stadtstatus konstatieren kann ${ }^{150}$.

\section{Fazit}

Die Untersuchung der acht württembergischen „Statuswüstungen“ Gönningen, Gutenberg, Heiningen, Hoheneck, Hohenhaslach, Horrheim, Neustadt und Ochsenburg hat eine Reihe von Beobachtungen und Erkenntnissen erbracht. Im Rahmen der württembergischen Urbanisierungsgeschichte wurde deutlich, dass der Verlust des städtischen Status nicht die Folge eines Rechtsaktes war bzw. auf eine gezielte Einwirkung seitens der Landes- und Stadtherren zurückging, sondern sich

146 Ähnlich erging es den „Statuswüstungen“ Weesen, Meienberg, Richensee und Rothenburg in der Nordschweiz: Ammann, Waadt (wie Anm. 8) S.76f.; Ammann, Kleinstadt (wie Anm. 8), S. 168 und 182; Stercken, Stadtstatus (wie Anm. 8).

147 Vgl. Schaав (wie Anm. 9) S. 223 und 225.

148 OAB Tübingen, S. 384; Kinkelin (wie Anm. 64) S. 11; LBW, Bd. 7, S. 64.

149 Küntzel (wie Anm. 9) S. 122, Schaubild 3; Ders. (wie Anm. 8) S. 38.

150 Vgl. zu den Ursachen für „Statuswüstungen“ insgesamt Ders. (wie Anm. 9) S.129, Schaubild 4; Eggert (wie Anm. 9) S. 228; Ammann, Waadt (wie Anm. 8) S. 79; Enders (wie Anm. 8) S. 117; Stephan (wie Anm. 9) S.330; Schaab (wie Anm. 9) S. 262; Küntzel (wie Anm. 8) S. 38, Abb. 3; Flückiger-Seiler (wie Anm. 8) S. 192; Engel (wie Anm. 4) S. 11. 
als vielschichtiger schleichender Prozess darstellt ${ }^{151}$. Diesem Prozess fielen solche Städte zum Opfer, die über strukturelle Defizite verfügten und diese infolge veränderter Rahmenbedingungen nicht mehr kompensieren konnten. Aus territorialpolitischen Gründen erworben, blieben sie innerhalb der Grafschaft Württemberg ohne Bedeutung und fungierten in der Folge meist nur als Pfand für Kreditgeber. Stellt man die Frage, was es genau war, was diese Städte verloren, was also den Unterschied zu einem bloßen Dorf ausmachte, so kann als Antwort nur auf ein Bündel von administrativen, wirtschaftlichen, rechtlichen und fortifikatorischen Funktionen verwiesen werden, die nur in ihrer Gesamtheit die Stadt konstituierten und sich einzeln nicht herausdeuten lassen. Im neuzeitlichen Württemberg allerdings war der Stadttitel vorrangig mit der Verwaltungskompetenz eines Ortes innerhalb des Ämtersystems verbunden, so dass sich hier wahrscheinlich ein begrifflicher Wandel vollzog.

In Bezug auf die „Statuswüstung“ als städtischer Übergangsform kristallisierte sich als grundsätzlich problematisch heraus, dass sich aufgrund der Quellenarmut nur schwer differenzieren lässt, ob eine Siedlung den Stadtstatus tatsächlich erreicht hatte, ehe er wieder verloren ging, oder ob die Stadtwerdung erst gar nicht zum Abschluss kam. So ist es etwa nicht eindeutig zu belegen, ob Heiningen tatsächlich vom Dorf zur Stadt aufstieg und dann zum Markt herabsank oder ob es sich nicht einfach nur vom Dorf zum Markt entwickelte ${ }^{152}$. Auch die städtische Qualität Ochsenburgs ist schon häufig angezweifelt worden ${ }^{153}$ und konnte hier so wenig nachgewiesen wie widerlegt werden. Der entscheidende Unterschied zu regulären Städten liegt dabei darin begründet, dass jene zwar häufig über ähnlich nebulöse, selbst durch eingehendes Quellenstudium nicht mehr greifbare Stadtwerdungsprozesse verfügen, jedoch zahlreiche Termini ante quos, meist in Form von Datensammlungen - die erste Nennung als Stadt, die erste Erwähnung städtischer Verwaltungsorgane, die ersten Überlieferungen zu Stadtsiegel und Stadtmauer -, letztlich keinen Zweifel am erfolgreichen Abschluss der städtischen Gründung aufkommen lassen. Diese markanten Punkte fehlen bei den kurzlebigen "Statuswüstungen" oft oder wurden vielleicht aufgrund der geringen Bedeutung dieser Siedlungen gar nicht erst schriftlich erfasst. Für die Analyse hatte dies zur Folge, dass viele Prozesse nur unter Vorbehalt beschrieben werden konnten und manches in der Schwebe bleibt. Abhilfe könnten hier nicht nur größer angelegte vergleichende Untersuchungen schaffen, sondern auch intensive Kooperationen mit der Stadtarchäologie, deren fruchtbare Ansätze sich unter anderem in den Arbeiten von Thomas Küntzel zeigen.

151 Anders hingegen Martina Stercken, die in der Nordschweiz Fälle „bewusster Deurbanisierung" ausgemacht hat: STERCKen, Städte (wie Anm. 8) S. 40.

152 Zur zweiten Variante s. Bauer (wie Anm. 40) S. 24.

153 S. z.B. Schанв (wie Anm. 9) S. 225. 